\title{
High-Energy Behavior of Total Scattering Cross Sections for 3-body Quantum Systems
}

\author{
By
}

Hiroshi T. ITO*

\section{§1. Introduction}

In this paper, we investigate the high-energy behavior of total scattering cross sections with 2-body initial states for a 3-body system.

A 3-body Schrödinger operator is given by

$$
\left.\widetilde{H}=-\sum_{1 \leqq j \leqq 3}\left(2 m_{\jmath}\right)^{-1}\right\lrcorner_{r_{\jmath}}+\sum_{1 \leqq i<j \geqq 3} V_{\imath \jmath}\left(r_{\imath}-r_{\jmath}\right) \quad \text { in } L^{2}\left(\boldsymbol{R}^{3 . v}\right) .
$$

Here $m_{\jmath}>0$ and $r_{\jmath} \in \boldsymbol{R}^{N^{*}}(N \geqq 3)$ are the mass and the position of the $j$-th particle, respectively, and $V_{1}$ is the interaction between the $i$-th particle and the $j$-th particle. All $V_{1}$ are real-valued functions and satisfy the following condition for some $\ell \in \boldsymbol{N} \backslash\{0\}$ or $\ell=3 / 2(\boldsymbol{N}=\{1,2, \cdots\})$ :

$$
V_{\imath \jmath}(x) \in C^{2 \ell+2}\left(\boldsymbol{R}^{v}\right) \text { and there exists a } \delta>\ell+((\nu+1) / 2)
$$

such that

$$
\left|\partial_{c}^{\gamma} V_{\imath \jmath}(x)\right| \leqq C\langle x\rangle^{-\delta}
$$

for all multi-indices $\gamma$ with $|\gamma| \leqq 2 \ell+2$, where $\langle x\rangle:=\left(1+|x|^{2}\right)^{1,2}$.

Let $H$ be the Schrödinger operator obtained by separating the kinetic energy of the center of mass from $\tilde{H} . H$ acts in $\mathscr{H}:=L^{2}\left(\boldsymbol{R}^{2 v}\right)$, and its explicit form depends on the coordinates of $\boldsymbol{R}^{2 .}$. We adopt the Jacobi coordinates. A partition of the set $\{1,2,3\}$ into nonempty disjoint subsets is called a cluster decomposition. We call $\{(1),(2),(3)\}$ (resp. $\{(i, j), k\}, i<j)$ a 3-cluster decomposition (resp. a 2-cluster decomposition). We denote by $\boldsymbol{A}_{2}$ the set of all 2-cluster decompositions. For $a \in \boldsymbol{A}_{2}$ with $a=\{(i, j), k\}$, we define the Jacobi coordinates $\left(x_{a}, y_{a}\right)$ by

$$
x_{a}=r_{1}-r_{\jmath}, \quad y_{a}=r_{k}-\frac{m_{\imath} r_{\imath}+m_{\jmath} r_{\jmath}}{m_{\imath}+m_{\jmath}} .
$$

Communicated by H. Araki, August 3, 1992.

1991 Mathematics Subject Classification: 81U10, 81U20, 35P25.

- Department of Mathematics, Kyoto University, Kyoto 606, Japan. 
Let $b \in \boldsymbol{A}_{2}$. Then $x_{b}, y_{b}$ are linear combinations of $x_{a}, y_{a}$. By the coordinates, $H$ is expressed as

$$
H=H_{0}+V=-\left(2 m_{a}\right)^{-1} \Delta_{x_{a}}-\left(2 n_{a}\right)^{-1} \Delta_{y_{a}}+V,
$$

where $V=\sum_{1 \leqq p<q \leqq 3} V_{p q}$ and $V_{p q}=V_{p q}\left(r_{p}-r_{q}\right)$. Note that $r_{p}-r_{q}$ is expressed as a linear combination of $x_{a}, y_{a} . m_{a}$ and $n_{a}$ are the reduced masses defined by $m_{a}^{-1}:=m_{\imath}^{-1}+m_{j}^{-1}, n_{a}^{-1}:=m_{k}^{-1}+\left(m_{\imath}+m_{\jmath}\right)^{-1}$, respectively.

Under assumption $(\mathrm{V})_{0}, H$ is self-adjoint with domain $D(H)=H^{2}\left(\boldsymbol{R}^{2 N}\right)$, the Sobolev space of order 2 .

For $a \in \boldsymbol{A}_{2}$ with $a=\{(i, j), k\}$, the cluster Hamiltonian $H_{a}$ is defined by

$$
H_{a}:=H_{0}+V_{\imath \jmath}, \quad D\left(H_{a}\right)=H^{2}\left(\boldsymbol{R}^{2 \mathrm{v}}\right) .
$$

$H_{a}$ is expressed as $H_{a}=h_{a} \otimes \operatorname{Id}+\operatorname{Id} \otimes T_{a}$ according to the decomposition $\mathscr{H}=$ $L^{2}\left(\boldsymbol{R}_{x_{a}}^{N}\right) \otimes L^{2}\left(\boldsymbol{R}_{y_{a}}^{N}\right)$, where $h_{a}:=-\left(2 m_{a}\right)^{-1} \Delta_{x_{a}}+V_{\imath \jmath}$ and $T_{a}:=-\left(2 n_{a}\right)^{-1} \Delta_{y_{a}}$ are self-adjoint in $L^{2}\left(\boldsymbol{R}_{x_{a}}^{N}\right)$ with $D\left(h_{a}\right)=H^{2}\left(\boldsymbol{R}_{x_{a}}^{N}\right)$ and in $L^{2}\left(\boldsymbol{R}_{y_{a}}^{N}\right)$ with $D\left(T_{a}\right)=$ $H^{2}\left(\boldsymbol{R}_{y_{a}}^{N}\right)$, respectively.

Let $d_{a}$ be the number of strictly negative bound state energies (counting multiplicity) of $h_{a}$. It is known that under assumption $(\mathrm{V})_{0}, d_{a}$ is finite (cf. [RS] IV, XIII. 3). We set the set of the 2-body channels with negative bound states energies:

$$
\Gamma_{2}:=\left\{\alpha=(a, k) ; a \in A_{2}, 1 \leqq k \leqq d_{a}, k \in \boldsymbol{N}\right\},
$$

and write $D(\alpha)=a$ for $\alpha=(a, k) \in \Gamma_{2}$. For each 2-body channel $\alpha=(a, k) \in \Gamma_{2}$ we denote by $\lambda_{\alpha}(<0)$ the $k$-th negative eigenvalue of $h_{a}$ and by $\psi_{\alpha}$ the eigenfunction of $h_{a}$ with eigenvalue $\lambda_{\alpha}$ such that $\left\{\psi_{\alpha}\right\}\left(\alpha \in \Gamma_{2}, D(\alpha)=a\right)$ is an orthonormal system for each $a \in \boldsymbol{A}_{2}$. For each $\alpha=(a, k) \in \Gamma_{2}$ the channel Hamiltonian $H_{a}$ in $L^{2}\left(\boldsymbol{R}_{y_{a}}^{N}\right)$ and the channel identification operator $J_{a} \in \boldsymbol{B}\left(L^{2}\left(\boldsymbol{R}_{y_{a}}^{N}\right), \mathscr{H}\right)$ are defined by

$$
H_{\alpha}=\lambda_{\alpha}+T_{a}, \quad J_{\alpha} u=\phi_{a} \otimes u,
$$

respectively. Here we denote by $\boldsymbol{B}(X, Y)$ the space of all bounded linear operators from $X$ to $Y$. Under assumption $(\mathrm{V})_{0}$ the channel wave operators:

$$
W_{\alpha}^{ \pm}:=s-\lim _{t \rightarrow \pm \infty} e^{i t H} J_{\alpha} e^{-i t H_{a}} \in \boldsymbol{B}\left(L^{2}\left(\boldsymbol{R}_{y_{a}}^{N}\right), \mathscr{H}\right)
$$

exist (see, e. g. [RS] III, Theorem XI. 35).

We set

$$
\Gamma:=\Gamma_{2} \cup\{0\},
$$

where $0 \in \Gamma$ stands for the 3-body channel.

For the 3-body channel the channel Hamiltonian is $H_{0}$ and the channel wave operators are defined by 


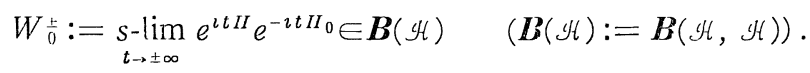

The existence of the channel wave operators is also known (see, e. g. [RS」III, Theorem XI. 35).

Throughout this paper we only consider the case where the initial channel belongs to $\Gamma_{2}$ and the inal channel belongs to $\Gamma$. The scattering operator $S_{a \rightarrow \beta}$ for scattering $\alpha \rightarrow \beta\left(\alpha \in \Gamma_{2}, \beta \in \Gamma\right)$ is defined by

$$
S_{a \rightarrow \beta}=W_{\beta}^{+*} W_{a}^{-},
$$

where $\Lambda^{*}$ denotes the adjoint of an operator 1 . Since the intertwining property, $\exp \left(i t H_{\beta}\right) S_{a \rightarrow \beta}=S_{\alpha \rightarrow \beta} \exp \left(i t H_{a}\right)$, holds, $S_{a \rightarrow \beta}$ is decomposable by a family of operators $\left\{S_{a \rightarrow \beta}(\lambda)\right\}, \lambda>\lambda_{\beta a}:=\max \left(\lambda_{a}, \lambda_{\beta}\right)$ (cf. [AJS], 15-3). The representation formula of $T_{a \rightarrow \beta}(\lambda):=S_{\alpha \rightarrow \beta}(\lambda)-\delta_{\beta \alpha}$ will be given in the next section and the Appendix, where $\delta_{\beta a}$ is Kronecker's delta and we set $\lambda_{\beta}=0$ if $\beta=0 . \quad T_{a \rightarrow \beta}(\lambda)$ is defined for a. e. $\lambda>\lambda_{\beta \alpha}$ as an operator in $\boldsymbol{B}\left(L^{2}\left(S^{N-1}\right), L^{2}\left(S^{3}\right)\right)$, where $S^{\beta}:=S^{N-1}$ (the unit sphere in $\boldsymbol{R}^{v}$ ) for $\beta \neq 0$ and $S^{3}=S^{2 N-1}$ for $\beta=0$.

If $\beta \neq 0, T_{a \rightarrow \beta}(\lambda)$ is well-defined as a norm continuous function of $\lambda>0$ and is of Hilbert-Schmidt class with kernel $T_{a \rightarrow \beta}(\lambda, \theta, \omega)$, and $T_{a \rightarrow \beta}(\lambda, \cdot, \omega)$ is $L^{2}\left(S^{v-1}\right)$-valued strongly continuous function of $\lambda>0$ and $\omega \in S^{v-1}$ (Proposition 2.3).

To treat the case $\beta=0$, we need the following condition in addition of $(\mathrm{V})_{0}$ :

(Z) For each $a=\{(i, j), k\} \in \boldsymbol{A}_{2}, \quad-1$ is not an eigenvalue of the following bounded operator on $L^{2}\left(\boldsymbol{R}_{x_{a}}^{N}\right)$ :

$$
\begin{aligned}
& V_{\imath \jmath}^{1 / 2}\left(-\left(2 m_{a}\right)^{-1} \Delta_{x_{a}}-0-i 0\right)^{-1}\left|V_{\imath \jmath}\right|^{1 / 2}, \\
(:= & \left.\lim _{\varepsilon \downarrow 0} V_{\imath \jmath}^{1 / 2}\left(-\left(2 m_{a}\right)^{-1} \Delta_{x_{a}}-0-i \varepsilon\right)^{-1}\left|V_{\imath \jmath}\right|^{1 / 2}\right),
\end{aligned}
$$

where $V_{\imath \jmath}^{1 / 2}:=\left|V_{\imath \jmath}\left(x_{a}\right)\right|^{1 / 2} \operatorname{sgn} V_{\imath \jmath}\left(x_{a}\right)$ and the existence of the norm limit is known (cf. [GM], Proposition 3.1)).

Assumption ( $Z$ ) implies the absence of zero eigenvalue of $h_{a}$ (cf. [GM], Proposition (3.4)), and assumption (V) implies the absence of positive eigenvalues (cf. [RS], XIII. 13). Therefore, it follows that the set of all eigenvalues of $h_{a}$ coincides with the set $\left\{\lambda_{\alpha} ; \alpha \in \Gamma_{2}, D(\alpha)=a\right\}$ under assumptions $(\mathrm{Z}),(\mathrm{V})_{0}$.

Under assumptions $(\mathrm{V})_{0}$ and $(Z), T_{a \rightarrow 0}(\lambda)$, is of Hilbert-Schmidt class with kernel $T_{\alpha \rightarrow 0}(\lambda, 0, \omega)$ for all large $\lambda>0$ and the integral

$$
\int_{S^{2 N-1}}\left|T_{\alpha \rightarrow 0}(\lambda, \theta, \omega)\right|^{2} d \theta
$$

is continuous in $\lambda \gg 1$ and $\boldsymbol{\omega} \in S^{N-1}$ (Proposition 2.4).

Now we give the following definition (see [AJS], p. 627): 
Definition. The total scattering cross section $\sigma_{\alpha \rightarrow \beta}(\lambda, \omega)$ for scattering $\alpha \rightarrow \beta\left(\alpha \in \Gamma_{2}, \beta \in \Gamma\right)$ at energy $\lambda \gg 1$ and incident direction $\omega \in S^{N-1}$ is delined by

$$
\sigma_{\alpha \rightarrow \beta}(\lambda, \omega):=(2 \pi)^{N-1}\left[2 n_{a}\left(\lambda-\lambda_{\alpha}\right)\right]^{\left(1-N^{j}\right) / 2} \int_{S \beta}\left|T_{\alpha \rightarrow \beta}(\lambda, \theta, \omega)\right|^{2} d \theta,
$$

under assumption $(\mathrm{V})_{0}$ for $\beta \neq 0$ and under assumptions $(\mathrm{V})_{0}$ and $(\mathrm{Z})$ for $\beta=0$. The total scattering csoss section for an initial channel $\alpha$ at energy $\lambda \gg 1$ and incident direction $\omega \in S^{N-1}$ is defined by

$$
\sigma_{a}(\lambda, \omega):=\sum_{\beta \in \Gamma} \sigma_{a-\beta}(\lambda, \omega)
$$

under assumptions $(\mathrm{V})_{0}$ and $(\mathrm{Z})$.

For $a=\{(i, j), k\} \in A_{2}$ we define the intercluster potential $I_{a}$ by

$$
I_{a}\left(x_{a}, y_{a}\right)=V-V_{1 \jmath}\left(x_{a}\right)
$$

and set

$$
W_{a}\left(x_{a} ; \omega, \eta\right):=\int_{R} I_{a}\left(x_{a}, t \omega+\eta\right) d t
$$

for $\omega \in S^{N-1}$ and $\eta \in \Pi_{\omega}:=\left\{\xi \in \boldsymbol{R}^{N} ; \xi \cdot \omega=0\right\} .(\cdot, \cdot)_{a}$ and $\|\cdot\|_{a}$ denote the $L^{2}$ scalar product and the $L^{2}$-norm in $L^{2}\left(\boldsymbol{R}_{x_{a}}^{N}\right)$, respectively.

Now we state our main results.

Theorem 1.1. Let $\alpha \in \Gamma_{2}(a=D(\alpha))$ and $\beta \in \Gamma$, and let the notations be as above.

(i) Assume $(\mathrm{V})_{\ell}$ with $\ell \in \boldsymbol{N} \cup\{0\}$ and $\beta \in \Gamma_{2}$ with $D(\beta) \neq a$. Then

$$
\sigma_{\alpha \rightarrow \beta}\left((1 / 2) n_{a} v^{2}+\lambda_{a}, \omega\right)=O\left(v^{-2 \ell-1}\right)
$$

uniformly in $\omega \in S^{N-1}$ as $v \rightarrow+\infty$.

(ii) Assume $(\mathrm{V})_{0}$ and $\beta \in \Gamma_{2}$ with $D(\beta)=a$. Then

$$
\sigma_{a \rightarrow \beta}\left((1 / 2) n_{a} v^{2}+\lambda_{a}, \omega\right)=v^{-2} \int_{\Pi_{\omega}}\left|\left(W_{a}(\cdot ; \omega, \eta) \psi_{\alpha}, \phi_{\beta}\right)_{a}\right|^{2} d \eta+o\left(v^{-2}\right)
$$

uniformly in $\omega \in S^{N-1}$ as $v \rightarrow+\infty$.

(iii) Assume $(\mathrm{V})_{0},(\mathrm{Z})$ and $\beta=0$. Then

$$
\begin{aligned}
\sigma_{\alpha \rightarrow \beta}\left((1 / 2) n_{a} v^{2}+\lambda_{a}, \omega\right) & =v^{-2} \int_{I_{\omega}}\left\|P^{c}\left(h_{a}\right) W_{a}(\cdot ; \omega, \eta) \psi_{a}\right\|_{a}^{2} d \eta+o\left(v^{-2}\right), \\
\sigma_{\alpha}\left((1 / 2) n_{a} v^{2}+\lambda_{\alpha}, \omega\right) & =v^{-2} \int_{\Pi_{\omega}}\left\|W_{\alpha}(\cdot ; \omega, \eta) \psi_{\alpha}\right\|_{a}^{2} d \eta+o\left(v^{-2}\right),
\end{aligned}
$$

uniformly in $\omega \in S^{N-1}$ as $v \rightarrow+\infty$, where $P^{c}\left(h_{a}\right)$ is the orthogonal projection onto the continuity subspace of $L^{2}\left(\boldsymbol{R}_{x_{a}}^{N}\right)$ with respect to $h_{a}$. 
Theorem 1.2. If we replace assumption $(\mathrm{V})_{0}$ by assumption $(V)_{3 / 2}$ in (ii), (iii) of the above theorem, all the remainder terms o( $\left.v^{-2}\right)$ in (1.19), (1.20), (1.21) can be replaced by $O\left(v^{-3}\right)$.

There are several literature on 3-body total cross sections ([APS], [ES], [AJS], [AS], [I], [IT]). In particular, bounds on the total cross sections at high energies for many-body systems are discussed in [APS], but the asymptotic behavior are not discussed in it. In [APS] and [ES] the approach to study the total cross sections is the time-dependent one, while our approach is the timeindependent one and is based on the representation formula of the scattering matrix and some resolvent estimates, which is proved by using multiple commutator methods ([JMP $]$ ). A similar approach is carried out in [I]. In [Ha] the convergence of Born series for (2-cluster) $\rightarrow$ (2-cluster) $T$-matrix for $n(\leqq 4)$ body systems at high energies is proved.

The organization of this paper is as follows. In Section 2 and in the Appendix, we shall review some properties of $T_{\alpha \rightarrow \beta}(\lambda, \theta, \omega)$ and prove the optical theorem (Theorem 2.5). The proof of Theorems 1.1 and 1.2 will be given in Section 3. A proof of Proposition 3.1, which is crucial for a proof of Theorem 1.1, will be given in Section 5 by using the abstract commutator methods (Theorem 4.2) in Section 4.

\section{§2. Representation Formula of $T_{a \rightarrow \beta}(\lambda, \theta, \omega)$}

In this section we will give the representation formula of $T_{\alpha \rightarrow \beta}(\lambda, \theta, \omega)$ for $\alpha \in \Gamma_{2}(a=D(\alpha))$ and $\beta \in \Gamma$, and will prove the optical theorem.

We first consider the case $\beta \in \Gamma_{2}$ with $b=D(\beta)$. The next lemmas are crucial for our representation formula of $T_{\alpha \rightarrow \beta}(\lambda, \theta, \omega)$. We write $R(z)=$ $(H-z)^{-1}$ for $\operatorname{Im} z \neq 0$.

Lemma 2.1 ([M], [PSS]). 1ssume $(V)_{0}$ and $s>1 / 2$. Then the norm limits

$$
R(\lambda \pm i 0):=\lim _{\varepsilon \downarrow 0} R(\lambda \pm i \varepsilon)
$$

exist in $\boldsymbol{B}\left(L_{s}^{2}\left(\boldsymbol{R}^{2 N}\right), L_{-s}^{2}\left(\boldsymbol{R}^{2 N}\right)\right)$ for $\lambda>0$, and the convergence is uniform on each compact subset in $(0, \infty)$, where $L_{t}^{2}\left(\boldsymbol{R}^{2 N}\right)(t \in \boldsymbol{R})$ is the weighted $L^{2}$-space:

$$
L_{t}^{2}\left(\boldsymbol{R}^{2 N}\right):=L^{2}\left(\boldsymbol{R}^{2 N} ;\left\langle x_{a} ; y_{a}\right\rangle^{2 t} d x_{a} d y_{a}\right),
$$

where $\left\langle x_{a} ; y_{a}\right\rangle:=\left(1+\left|x_{a}\right|^{2}+\left|y_{a}\right|^{2}\right)^{1 / 2}$. (Since $m_{a}\left|x_{a}\right|^{2}+n_{a}\left|y_{a}\right|^{2}=m_{b}\left|x_{b}\right|^{2}+$ $n_{b}\left|y_{b}\right|^{2}$ for any $b \in \boldsymbol{A}_{2}$, the definition of the space $L_{l}^{2}\left(\boldsymbol{R}^{2 N}\right)$ is independent of the choice of $a \in A_{2}$.)

Lemma 2.2. Assume $(\mathrm{V})_{\iota}$. Then for any $s>0$ and any multi-index $\gamma$ with $|\gamma| \leqq 2 \ell+4, \psi_{\beta}$ satisfies $\partial_{r_{b}}^{r_{\beta}} \psi_{\beta} \in L_{s}^{2}\left(\boldsymbol{R}_{x_{b}}^{N}\right)$. 
For the proof of Lemma 2.1, see [PSS]. Lemma 2.1 (called the limiting absorption principle) holds for $n(\geqq 2)$-body systems under milder conditions on the potentials $([\mathrm{M}]$, [PSS $],[A B G],[T])$. Lemma 2.2 is known as the exponential decay of eigenfunctions. For the proof, see [Ag] (see also [RS] IV, XIII. 11). Now we give the spectral representation of $H_{\beta}$ (cf. [AJS], 16-2).

For $\lambda>\lambda_{\beta}$ we define a map $Z_{\beta}(\lambda) \in \boldsymbol{B}\left(L_{s}^{2}\left(\boldsymbol{R}_{y_{b}}^{N}\right), L^{2}\left(S^{N-1}\right)\right), s>1 / 2$, by

$$
\left(Z_{\beta}(\lambda) f\right)(\omega)=C_{\beta}(\lambda) \int e^{-i\left(2 n_{b}(\lambda-\lambda \beta)\right)^{1 / 2} \omega \cdot y_{b}} f\left(y_{b}\right) d y_{b},
$$

where $\omega \in S^{N-1}$ and

$$
C_{\beta}(\lambda)=(2 \pi)^{-N / 2} n_{b}^{1 / 2}\left(2 n_{b}\left(\lambda-\lambda_{\beta}\right)\right)^{(N-2) / 4} .
$$

Then the map $Z_{\beta}$, defined by

$$
\left(Z_{\beta} f\right)(\lambda, \omega)=\left(Z_{\beta}(\lambda) f\right)(\omega),
$$

can be extended to a unitary operator from $L^{2}\left(\boldsymbol{R}_{y_{b}}^{N}\right)$ to $L^{2}\left(\left(\lambda_{\beta}, \infty\right) ; L^{2}\left(S^{N-1}\right)\right)$ and

$$
\left(Z_{\beta} H_{\beta} f\right)(\lambda, \cdot)=\lambda\left(Z_{\beta} f\right)(\lambda, \cdot)
$$

in $L^{2}\left(S^{N-1}\right)$ for a. e. $\lambda>\lambda_{\beta}$ if $f \in D\left(H_{\beta}\right) . \quad Z_{\alpha}$ is defined in the same way.

We define $G_{\beta \alpha}(\lambda, \omega)=G_{\beta \alpha}\left(\lambda, \omega ; y_{b}\right)$ by

$$
G_{\beta \alpha}(\lambda, \omega)=\int \overline{\phi_{\beta}\left(x_{b}\right)}\left(K(\lambda) e_{a}(\lambda, \omega)\right)\left(x_{b}, y_{b}\right) d x_{b},
$$

where $K(\lambda)=-I_{a}+I_{b} R(\lambda+i 0) I_{a}$, and

$$
e_{\alpha}(\lambda, \omega)=\phi_{\alpha}\left(x_{a}\right) e^{i\left(2 n_{a}\left(\lambda-\lambda_{a}\right)\right) 1 / 2_{\omega} \cdot y_{a}} .
$$

From $(\mathrm{V})_{0}$ and Lemmas 2.1, 2.2, it follows that $G_{\beta \alpha}(\lambda, \omega)$ is $L_{s}^{2}\left(\boldsymbol{R}_{y_{b}}^{N}\right)$-valued strongly continuous function of $(\lambda, \omega) \in(0, \infty) \times S^{v-1}$ if $1 / 2<s<\delta-(N / 2)$.

Proposition 2.3. Let $\alpha, \beta \in \Gamma_{2}$ and assume $(\mathrm{V})_{0}$. Then $Z_{\beta} S_{\alpha \rightarrow \beta} Z_{\alpha}^{*}$ is decomposable by a family of operators $\left\{S_{\alpha \rightarrow \beta}(\lambda)\right\}, \lambda>0$ :

$$
\left(Z_{\beta} S_{\alpha \rightarrow \beta} Z_{\alpha}^{*} h\right)(\lambda)=S_{\alpha \rightarrow \beta}(\lambda) h(\lambda) \quad \text { in } L^{2 /}\left(S^{N-1}\right)
$$

for a.e. $\lambda>0$, where $h \in L^{2}\left((0, \infty) ; L^{2}\left(S^{N-1}\right)\right)$, which is considered to be embedded in $L^{2}\left(\left(\lambda_{\alpha}, \infty\right) ; L^{2}\left(S^{N-1}\right)\right)$ by regarding $h(\lambda)=0$ for $\lambda \in\left(\lambda_{\alpha}, 0\right]$. Furthermore, $T_{a \rightarrow \beta}(\lambda):=S_{\alpha \rightarrow \beta}(\lambda)-\delta_{\alpha \beta} \in \boldsymbol{B}\left(L^{2}\left(S^{N-1}\right)\right)$ is continuous in $\lambda>0$ with respect to the Hilbert-Schmidt norm and its kernel $T_{\alpha \rightarrow \beta}(\lambda, \theta, \omega), \theta, \omega \in S^{N-1}$, is given by

$$
T_{\alpha \rightarrow \beta}(\lambda, \theta, \omega)=2 \pi i C_{\alpha}(\lambda)\left(Z_{\beta}(\lambda) G_{\beta \alpha}(\lambda, \omega)\right)(\theta) .
$$

In particular, $T_{\alpha \rightarrow \beta}(\lambda, \cdot, \omega)$ is $L^{2}\left(S^{N-1}\right)$-valued continuous function of $(\lambda, \omega)$ and the kernel $\left(\operatorname{Re} T_{\alpha \rightarrow \alpha}(\lambda)\right)(\theta, \omega)$ of $(1 / 2)\left(T_{\alpha \rightarrow \alpha}(\lambda)+T_{\alpha \rightarrow \alpha}(\lambda)^{*}\right)$ is continuous in $(\lambda, \theta, \omega)$.

Proof. The first half of this proposition and (2.6) can be proved in almost 
the same way as in the 2-body case. For the proof of the first half, see, for example, Proposition 2.4 of [1]. (2.6) yields

$$
\left(\operatorname{Re} T_{\alpha \rightarrow \alpha}(\lambda)\right)(\theta, \omega)=\pi i C_{\alpha}(\lambda)^{2}\left((R(\lambda+i 0)-R(\lambda-i 0)) I_{a} e_{a}(\lambda, \omega), I_{a} e_{\alpha}(\lambda, \theta)\right) .
$$

We fix $s$ with $1 / 2<s<\delta-(N / 2)$. Then, by $(\mathrm{V})_{0}$ and Lemma $2.2, I_{a} e_{\alpha}(\lambda, \omega)$ is $L_{s}^{2}\left(\boldsymbol{R}^{2 N}\right)$-valued strongly continuous function of $(\lambda, \omega)$. Thus, the last half of the proposition follows from $(2.6)^{\prime}$ and Lemma 2.1 .

We next consider the case $\beta=0$. To give the spectral representation of $H_{0}$, we define a unitary operator $U$ on $\mathscr{H}$ by

$$
(U f)\left(x_{a}, y_{a}\right)=\left(2 m_{a}\right)^{-N / 4}\left(2 n_{a}\right)^{-N / 4} f\left(\left(2 m_{a}\right)^{-1 / 2} x_{a},\left(2 n_{a}\right)^{-1 / 2} y_{a}\right)
$$

and define an operator $Z_{0}(\lambda) \in \boldsymbol{B}\left(L_{s}^{2}\left(\boldsymbol{R}^{2 N}\right), L^{2}\left(S^{2 N-1}\right)\right), \lambda>0, s>1 / 2$, by

$$
\left(Z_{0}(\lambda) f\right)(\theta)=C_{0}(\lambda) \int e^{-i \lambda 1 / 2 \theta \cdot X}(U f)(X) d X,
$$

where $\theta \in S^{2 N-1}, X=\left(x_{a}, y_{a}\right), d X=d x_{a} d y_{a}$ and

$$
C_{0}(\lambda)=2^{-N / 2}(2 \pi)^{-N}(2 \lambda)^{(N-1) / 2} .
$$

Then the map $Z_{0}$, defined by

$$
\left(Z_{0} f\right)(\lambda, \theta)=\left(Z_{0}(\lambda) f\right)(\theta),
$$

can be extended to a unitary operator from $\mathscr{K}$ to $L^{2}\left((0, \infty) ; L^{2}\left(S^{2 N-1}\right)\right)$ and give the spectral representation of $H_{0}$ : For each $f \in D\left(H_{0}\right)$,

$$
\left(Z_{0} H_{0} f\right)(\lambda, \cdot)=\lambda\left(Z_{0} f\right)(\lambda, \cdot) \quad \text { in } L^{2}\left(S^{2 N-1}\right)
$$

for a. e. $\lambda>0$.

Proposition 2.4. Assume $(\mathrm{V})_{0},(Z)$ and $\alpha \in \Gamma_{2}, \beta=0$. Then, $Z_{0} S_{\alpha \rightarrow 0} Z_{\alpha}{ }^{*}$ is decomposable by a family of operators $\left\{T_{\alpha \rightarrow 0}(\lambda)\right\}, \lambda>0 . \quad T_{\alpha \rightarrow 0}(\lambda) \in \boldsymbol{B}\left(L^{2}\left(S^{N-1}\right)\right.$, $\left.L^{2}\left(S^{2 N-1}\right)\right)$ is continuous in $\lambda \gg 1$ with respect to the Hilbert-Schmidt norm. Let $T_{\alpha \rightarrow 0}(\lambda, \theta, \omega), \theta \in S^{2-1}, \omega \in S^{N-1}$, be its integral kernel. Then, $T_{n \rightarrow 0}(\lambda, \cdot, \omega)$ is $L^{2}\left(S^{2 N-1}\right)$-valued continuous function of $\lambda \gg 1$ and $\omega \in S^{N-1}$.

Most of these results are obtained in [AS]. In [AS], it is shown that $T_{\alpha \rightarrow \beta^{\prime}}(\lambda)$ can be defined for all $\lambda>0$ but a closed null set, while the boundedness of this set is not proved in it. Thus, for completeness, we give a proof of Proposition 2.4 in the Appendix.

Owing to the following theorem, we need not study directly the asymptotic behavior of $\sigma_{a \rightarrow 0}(\lambda, \omega)$ as $\lambda \rightarrow \infty$.

Theorem 2.5. Assume $(\mathrm{V})_{0},(\mathrm{Z})$ and $\alpha \in \Gamma_{2}$. Then, for each $\lambda \gg 1$ and $\omega \in$ $S^{v-1}$, the following relation holds: 


$$
\sigma_{\alpha}(\lambda, \omega)=-2(2 \pi)^{N-1}\left(2 n_{a}\left(\lambda-\lambda_{\alpha}\right)\right)^{(1-N) / 2}\left(\operatorname{Re} T_{a \rightarrow \alpha}(\lambda)\right)(\omega, \omega)
$$

$(\operatorname{see}(1.15))$.

Proof. Under assumption (V), it is known that for $\alpha \neq \beta(\alpha, \beta \in \Gamma)$, Ran $W_{\alpha}^{-}$is orthogonal to Ran $W_{\beta}^{ \pm}$(Ran=Range) and the asymptotic completeness holds (see (1.8), (1.10)). Moreover, if we assume $(Z)$ as well, we have

$$
\sum_{\alpha \in \Gamma} \oplus \operatorname{Ran} W_{\alpha}^{ \pm}=\mathscr{K}_{a c}(H),
$$

where $\mathscr{H}_{a c}(H)$ denotes the absolute continuity subspace of $\mathscr{H}^{\prime}$ with respect to $H$ (cf. [E]). This yields

$$
\sum_{\beta \in \Gamma} S_{\alpha \rightarrow \beta} * S_{\alpha \rightarrow \beta}=\mathrm{Id}
$$

and so

$$
\sum_{\beta \in \Gamma} T_{n \rightarrow \beta}(\lambda)^{*} T_{\alpha, \beta}(\lambda)=-T_{n-n}(\lambda)-T_{n \rightarrow n}(\lambda)^{*} .
$$

This equality holds for each $\lambda \gg 1$ since all terms are continuous by Propositions 2.3 and 2.4. From (2.13) we have

$$
\sum_{\beta \in \Gamma} \int_{S \beta^{\beta}} \overline{T_{\kappa+\beta}(\lambda, \theta,} \overline{\left.\omega^{\prime}\right)} T_{\alpha \rightarrow \beta}(\lambda, \theta, \omega) d \theta=-2\left(\operatorname{Re} T_{n \rightarrow n}(\lambda)\right)\left(\omega^{\prime}, \omega\right)
$$

This equality holds for each $\omega, \omega^{\prime} \in S^{N-1}$ and $\lambda \gg 1$ since both sides are continuous by Propositions 2.3 and 2.4. Hence, putting $\omega^{\prime}=\omega$, we get the desired result.

Remark. This theorem is called the optical theorem ([AJS $],$ p. 628). We can show that $\sigma_{\alpha}(\lambda, \omega)$ is well-defined for a. e. $(\lambda, \omega) \in(0, \infty) \times S^{N-1}$ and $(2.11)$ holds for a.e. $(\lambda, \omega) \in(0, \infty) \times S^{N-1}$ under assumptions $(\mathrm{V})_{0}$ without $(Z)$ (see Proposition 2.3 in $\left[\mathrm{I}^{\prime} T\right]$ ).

\section{$\S 3$. Proof of Theorem 1.1}

3.1. In this subsection we assume $(\mathrm{V})_{\ell}$ with $\ell \in \boldsymbol{N} \cup\{0\}$. Let $\alpha, \beta \in \Gamma_{2}$ with $D(\alpha)=a, D(\beta)=b, a=b$. We write $x=x_{a}, y=y_{a}$ for simplicity. For $v>0$ we define

$$
\lambda(v):=(1 / 2) n_{a} v^{2}+\lambda_{\alpha} .
$$

If $v$ is large, we can take $v^{\prime}=v^{\prime}(v)>0$ such that

$$
(1 / 2) n_{b} v^{2}+\lambda_{\beta}=\lambda(v) \text {. }
$$

Throughout this section, we assume $\imath \gg 1$. By (2.4) and (2.6) we have

$$
T_{a \rightarrow \beta}(\lambda(v), \theta, \omega)=C(v)\left(\left[-I_{a}+I_{b} R(\lambda+i 0) I_{a}\right]\left(\psi_{a} e^{i n_{a} v(a \cdot y}\right), \psi_{\beta} e^{i n_{b} v^{\prime} \theta \cdot y_{b}}\right),
$$


where $\psi_{a}=\psi_{n}(x), \psi_{\beta}=\psi_{\beta}\left(x_{b}\right)$ and

$$
C(v):=i(2 \pi)^{-N+1}\left(n_{a} n_{b}\right)^{(N-1) / 2}\left(v v^{\prime}\right)^{(N-2) / 2} .
$$

Here notice that the R.H.S. of (3.3) is well-defined for all $(\lambda, \omega, \theta) \in(0, \infty) \times$ $S^{N-1} \times S^{N-1}$ by $(V)$, and $a \neq b$.

For each $v \gg 1$ and $\omega \in S^{N-1}$, we define a self-adjoint operator $L(v, \boldsymbol{\omega})$ in $\mathscr{H}$ by

$$
L(v, \omega):=v^{-1}\left(H-\lambda_{a}\right)-i \omega \cdot \Gamma_{y}=e^{-i n a v \omega \cdot y} v^{-1}(H-\lambda(v)) e^{i n a v \omega \cdot y} .
$$

By (3.5) and Lemma 2.1, the norm limit

$$
(L(v, \boldsymbol{\omega})-i 0)^{-1}:=\lim _{\varepsilon \downarrow 0}(L(v, \boldsymbol{\omega})-i \varepsilon)^{-1} \in \boldsymbol{B}\left(L_{s}^{2}\left(\boldsymbol{R}^{2 N}\right), L_{-s}^{2}\left(\boldsymbol{R}^{2 N}\right)\right)
$$

exists for $s>1 / 2$. Then $T_{a \rightarrow \beta}(\lambda(v), \theta, \omega)$ is written as

$$
\begin{aligned}
& T_{\alpha \rightarrow \beta}(\lambda(v), \theta, \omega) \\
& \quad=C(v)\left(\left[-I_{a}+v^{-1} I_{b}(L(v, \omega)-i 0)^{-1} I_{a}\right] \psi_{a}, \psi_{\beta} e^{i n b^{v^{\prime}} \theta \cdot y_{b^{-i n}} v(\omega \cdot y}\right) .
\end{aligned}
$$

For $k, s \in \boldsymbol{R}$ we define

$$
H_{s}^{k}\left(\boldsymbol{R}^{2 v}\right)=\left\{f \in \mathcal{S}^{\prime}\left(\boldsymbol{R}^{2 . v}\right) ;\|f\|_{k, s}:=\left\|\langle x ; y\rangle^{s}(-\Delta+1)^{k / 2} f\right\|_{L^{2}\left(R^{2 N}\right)}<\infty\right\},
$$

where $\mathcal{S}^{\prime}$ is the tempered distributions and $\Delta$ is the $2 N$-dimensional Laplacian.

Instead of $(\mathrm{V})_{\iota}$, we assume the following condition $(\mathrm{U})_{\iota}, \ell \in \boldsymbol{N} \cup\{0\}$, to prove the next proposition:

(U), Each $V_{\imath j}(x)(1 \leqq i<j \leqq 3)$ is a real-valued $C^{2 \iota+2}$-function on $\boldsymbol{R}^{N}$ and satisfies

$$
\left|D_{x}^{r} V_{\imath \jmath}(x)\right| \leqq C\langle x\rangle^{-\min (|\gamma|, \ell+2)}, \quad|\gamma| \leqq 2 \ell+2 .
$$

Since $N \geqq 3$, it is obvious that $(\mathrm{V})$, implies $(\mathrm{U})$, for $\ell \in \boldsymbol{N} \cup\{0\}$. The following proposition will be proved in Section 5 .

Proposition 3.1. (i) Assume $(\mathrm{U})_{\text {, }}$ and let $k$ be an integer with $0 \leqq k \leqq \ell$ and $s$ a real with $k+(1 / 2)<s$. Then there exists $a \quad v_{0}>0$ such that

$$
\sup _{\substack{0<\varepsilon<1, v \geq v_{0} \\ \omega \in S N N-1}}\left\|(L(v, \omega)-i \varepsilon)^{-1}\right\|_{\boldsymbol{B}_{k, s}}<\infty
$$

and the norm limit

$$
(L(v, \boldsymbol{\omega})-i 0)^{-1}:=\lim _{\varepsilon \downarrow 0}(L(v, \boldsymbol{\omega})-i \boldsymbol{\varepsilon})^{-1}
$$

exists in $\boldsymbol{B}_{k, s}:=\boldsymbol{B}\left(H_{s}^{k}\left(\boldsymbol{R}^{2 N}\right), H_{-s}^{k}\left(\boldsymbol{R}^{2 N}\right)\right)$ uniformly in $v \geqq v_{0}$ and $\boldsymbol{\omega} \in S^{N-1}$. In particular, the operator norm $\left\|(L(v, \omega)-i 0)^{-1}\right\|_{\boldsymbol{B}_{k} s}$ is uniformly bounded in $v \geqq v_{0}$ and $\omega \in S^{N-1}$.

(ii) Assume $(U)_{1}$. Then 


$$
\left\|(L(v, \omega)-i 0)^{-1}-\left(-i \omega \cdot \nabla_{y}-i 0\right)^{-1}\right\|_{\boldsymbol{B}\left(H_{2}^{4}, L_{-2}^{2}\right)}=O\left(v^{-1}\right)
$$

uniformly in $\boldsymbol{\omega} \in S^{N-1}$ as $v \rightarrow+\infty$ where $H_{2}^{4}=H_{2}^{4}\left(\boldsymbol{R}^{2 N}\right), L_{-2}^{2}=L_{-2}^{2}\left(\boldsymbol{R}^{2 N}\right)$

Remark. The norm limit

$$
\left(-i \omega \cdot \nabla_{y}-i 0\right)^{-1}:=\lim _{\varepsilon \downarrow 0}\left(-i \omega \cdot \nabla_{y}-i \varepsilon\right)^{-1} \in \boldsymbol{B}_{0, s}, \quad s>1 / 2,
$$

exists uniformly in $\omega \in S^{N-1}$ and

$$
\left(\left(-i \omega \cdot \nabla_{y}-i 0\right)^{-1} u\right)(x, \eta+t \omega)=i \int_{-\infty}^{t} u(x, \eta+s \omega) d s,
$$

where $\eta \in \Pi_{\omega}=\left\{\eta \in \boldsymbol{R}^{N} ; \eta \cdot \omega=0\right\}$. Indeed, in one dimensional case, $\left.\langle q\rangle^{-s}(-i(d / d q)-i \varepsilon)^{-1}\langle q\rangle^{-s}, \varepsilon>0, s\right\rangle 1 / 2$, is an integral operator with HilbertSchmidt kernel $K(\varepsilon ; q, t)=i\langle q\rangle^{-s} \exp (\varepsilon(t-q)) \chi(t, q)\langle t\rangle^{-s}$, where $\chi(t, q)=1$ (resp. $=0)$ for $t \leqq q$ (resp. $q \leqq t)$, and converges to a Hilbert-Schmidt operator with kernel $K(+0 ; q, t)=i\langle q\rangle^{-s} \chi(t, q)\langle t\rangle^{-s}$ w. r. t. the Hilbert-Schmidt norm as $\varepsilon \downarrow 0$.

Proof of Theorem 1.1 (i). Since $a \neq b, y$ is written as $y=m x_{b}+n y_{b}$ for some constants $m \neq 0, n \neq 0$. Thus, by (3.7), we have

$$
\begin{aligned}
& T_{\alpha \rightarrow \beta}(\lambda(v), \theta, \omega) \\
& =C(v) \int e^{\left(-i n_{b} v^{\prime} \theta+i n_{a} v n \omega\right) \cdot y_{b}} d y_{b} \int e^{i n_{a} v m \omega \cdot x_{b}}\left(f_{1}+v^{-1} f_{2}\right) d x_{b},
\end{aligned}
$$

where

$$
\begin{aligned}
& f_{1}=f_{1}\left(x_{b}, y_{b}\right)=-\bar{\psi}_{\beta}\left(x_{b}\right) I_{a} \psi_{\alpha}(x), \\
& f_{2}=f_{2}\left(x_{b}, y_{b}\right)=\bar{\psi}_{\beta}\left(x_{b}\right)\left(\left[I_{b}(L(v, \omega)-i 0)^{-1} I_{a}\right] \psi_{\alpha}\right)\left(x_{b}, y_{b}\right) .
\end{aligned}
$$

By $(\mathrm{V})_{\iota}$, Lemma 2.2 and Proposition 3.1, the following estimates can be verified:

$$
\begin{gathered}
\partial_{x_{b}}^{r_{1}} \partial_{y_{b}}^{r_{2}} f_{1} \in L^{1}\left(\boldsymbol{R}^{2 N}\right), \quad\left|\gamma_{1}\right|+\left|\gamma_{2}\right| \leqq \ell+1, \\
\sup _{v \gg 1, \omega \in S^{N-1}}\left\|\partial_{x_{b}}^{r_{1}} \partial_{y_{b}}^{r_{2}} f_{2}\right\|_{L^{1}\left(R^{2}\right)}<\infty, \quad\left|\gamma_{1}\right|+\left|\gamma_{2}\right| \leqq \ell, \\
\left(\sup _{v \gg 1} \cdots:=\sup _{v \gtrless v_{1}} \cdots \quad \text { for some large } v_{1}\right) .
\end{gathered}
$$

Hence, by integration by part in $x_{b}$, we can write

$$
T_{\alpha \rightarrow \beta}(\lambda(v), \theta, \omega)=C(v) v^{-\ell-1}\left(F_{y_{b}} g\right)\left(n_{b} v^{\prime} \theta-n_{a} v n \omega\right),
$$

where $\left(F_{y_{b}} g\right)(\xi)$ is the Fourier transform of

$$
g\left(y_{b}\right)=\int e^{i n_{a} v m \omega \cdot x_{b}} h\left(v, \omega ; x_{b}, y_{b}\right) d x_{b},
$$

where

$$
h=(2 \pi)^{N / 2}\left\{\left(i / n_{a} m\right)^{\ell+1}\left(\boldsymbol{\omega} \cdot \nabla_{x_{b}}\right)^{\ell+1} f_{1}+\left(i / n_{a} m\right)^{\ell}\left(\boldsymbol{\omega} \cdot \nabla_{x_{b}}\right)^{\ell} f_{2}\right\} .
$$

In the same way as (3.13), we have 


$$
\sup _{v \gg 1, \omega \in S^{N-1}}\left\|\left\langle x_{b}\right\rangle^{N}\left\langle y_{b}\right\rangle h(v, \omega ; \cdot, \cdot)\right\|_{L^{2}\left(R^{2} N\right)}<\infty .
$$

Thus, by (1.14), (3.14), we have

$$
\begin{aligned}
\sigma_{\alpha \rightarrow \beta}(\lambda(v), \omega)= & (2 \pi)^{N-1}\left(n_{a} n_{b} v v^{\prime}\right)^{1-N} C(v)^{2} v^{-2 \ell-2} \\
& \times \int_{|\xi|=n_{b} v^{\prime}}\left|\left(F_{y_{b}} g\right)\left(\xi-n_{a} v n \omega\right)\right|^{2} d S_{\xi},
\end{aligned}
$$

where $d S_{\xi}$ is the Lebesgue measure on the sphere $\left\{\xi \in \boldsymbol{R}^{N} ;|\xi|=n_{b} v^{\prime}\right\}$. On the other hand, by the trace theorem ([GM], Proposition 2.1) we get

$$
\begin{aligned}
\int_{|\xi|=n_{b} v^{\prime}}\left|\left(F_{y_{b}} g\right)\left(\xi-n_{a} v n \omega\right)\right|^{2} d S_{\xi} & \leqq C\left\|\left(F_{y_{b}} g\right)\left(\cdot-n_{a} v n \omega\right)\right\|_{H^{1}\left(R^{N}\right)}^{2} \\
& \leqq C\|g\|_{L_{1}^{2}\left(R_{y_{b}}^{N}\right)}^{2} \\
& \leqq C\left\|\left\langle x_{b}\right\rangle^{N}\left\langle y_{b}\right\rangle h(v, \omega ; \cdot, \cdot)\right\|_{L^{2}\left(R^{2}\right)}^{2},
\end{aligned}
$$

where we have used the Schwarz inequality in the last step. Therefore, by (3.4), (3.16) and (3.17), we obtain (1.18).

3.2. In this subsection we assume $(\mathrm{V})_{0}$. We begin with the following lemma.

Lemma 3.2. Let $\alpha, \beta \in \Gamma_{2}$ with $D(\alpha)=a, D(\beta)=b$. Then, for each $\lambda>0$ and each $\omega \in S^{N-1}, \sigma_{\alpha \rightarrow \beta}(\lambda, \omega)$ can be represented as

$$
\sigma_{\alpha \rightarrow \beta}(\lambda, \omega)=2(2 \pi)^{N} C_{\alpha}(\lambda)^{2}\left(2 n_{a}\left(\lambda-\lambda_{\alpha}\right)\right)^{(1-N) / 2} I(\lambda, \omega),
$$

where

$I(\lambda, \omega):=\operatorname{Im}\left(E_{\beta} R(\lambda+i 0) I_{a} e_{\alpha}, I_{a} e_{\alpha}\right)+\operatorname{Im}\left(E_{\beta} I_{b} R(\lambda+i 0) I_{a} e_{\alpha}, R(\lambda+i 0) I_{a} e_{\alpha}\right)$, $e_{\alpha}:=\phi_{\alpha}(x) e^{i\left(2 n_{a}\left(\lambda-\lambda_{\alpha}\right)\right)^{1 / 2} \omega \cdot y}$, and $E_{\beta}=J_{\beta} J_{\beta}^{*}(\operatorname{see}(1.7))$.

Remark. $I(\lambda, \omega)$ is well-defined by Lemma 2.1 and the following:

$$
\begin{gathered}
\langle x ; y\rangle^{s} I_{a} e_{\alpha} \in L^{2}\left(\boldsymbol{R}^{2 N}\right), \\
\langle x ; y\rangle^{-s} E_{\beta}\langle x ; y\rangle^{s} \in \boldsymbol{B}\left(L^{2}\left(\boldsymbol{R}^{2 N}\right)\right), \\
\langle x ; y\rangle^{s} E_{\beta} I_{b}\langle x ; y\rangle^{s} \in \boldsymbol{B}\left(L^{2}\left(\boldsymbol{R}^{2 N}\right)\right),
\end{gathered}
$$

for some $s>1 / 2$, which follow from $(\mathrm{V})_{0}$ and Lemma 2.2.

Proof. We first note that

$$
T_{a \rightarrow \beta}(\lambda, \theta, \omega)=2 \pi i C_{\alpha}(\lambda)\left(Z_{\beta}(\lambda) J_{\beta} * K(\lambda) e_{\alpha}\right)(\theta),
$$

where $K(\lambda):=-I_{a}+I_{b} R(\lambda+i 0) I_{a}$ (see (2.4), (2.6)). Thus we have 


$$
\begin{aligned}
\sigma_{n \rightarrow \beta}(\lambda, \omega)= & (2 \pi)^{N+1} C_{\alpha}(\lambda)^{2}\left(2 n_{\alpha}\left(\lambda-\lambda_{\alpha}\right)\right)^{(1-N) / 2} \\
& \times\left(Z_{\beta}(\lambda) * Z_{\beta}(\lambda) J_{\beta}^{*} K(\lambda) e_{\alpha}, J_{\beta}^{*} K(\lambda) e_{\alpha}\right) .
\end{aligned}
$$

Here we regard $Z_{\beta}(\lambda)^{*} \in \boldsymbol{B}\left(L^{2}\left(S^{N-1}\right), L_{-s}^{2}\left(\boldsymbol{R}_{y_{b}}^{N}\right)\right), s>1 / 2$, by regarding $L_{-s}^{2}$ as $\left(L_{s}^{2}\right)^{*}$. Therefore we get

$$
\begin{aligned}
\sigma_{\alpha \rightarrow \beta}(\lambda, \omega)= & -i(2 \pi)^{N} C_{\alpha}(\lambda)^{2}\left(2 n_{\alpha}\left(\lambda-\lambda_{a}\right)\right)^{(1-N) / 2} \\
& \times\left(\left[R_{b}(\lambda+i 0)-R_{b}(\lambda-i 0)\right] E_{\beta} K(\lambda) e_{\alpha}, K(\lambda) e_{a}\right),
\end{aligned}
$$

where $R_{b}(\lambda \pm i 0)=\left(H_{b}-(\lambda \pm i 0)\right)^{-1}:=\lim _{\varepsilon \downarrow 0}\left(H_{b}-(\lambda \pm i \varepsilon)\right)^{-1}$ (see (1.5), Lemma 2.1) is a bounded operator from $L_{s}^{2}\left(\boldsymbol{R}^{2 N}\right)$ to $L_{-s}^{2}\left(\boldsymbol{R}^{2 N}\right), s>1 / 2$, and we have used the following two relations in the last step:

$$
\begin{gathered}
Z_{\beta}(\lambda) * Z_{\beta}(\lambda)=(2 \pi i)^{-1}\left\{\left(H_{\beta}-(\lambda+i 0)\right)^{-1}-\left(H_{\beta}-(\lambda-i 0)\right)^{-1}\right\}, \\
J_{\beta}\left(H_{\beta}-(\lambda \pm i 0)\right)^{-1}=R_{b}(\lambda \pm i 0) J_{\beta} .
\end{gathered}
$$

Furthermore, the resolvent equation $R_{b}(z) I_{b} R(z)=R_{b}(z)-R(z)$ yields the following :

$$
R_{b}(\lambda+i 0) E_{\beta} K(\lambda)=-E_{\beta} R(\lambda+i 0) I_{a} .
$$

Thus, by this together with $R_{b}(\lambda+i 0) E_{\beta}=E_{\beta} R_{b}(\lambda+i 0)$, we can get the following relation:

$$
\begin{aligned}
K(\lambda) *\left[R_{b}(\lambda\right. & \left.+i 0)-R_{b}(\lambda-i 0)\right] E_{\beta} K(\lambda) \\
= & I_{a} E_{\beta} R(\lambda+i 0) I_{a}-I_{a} R(\lambda-i 0) E_{\beta} I_{a} \\
& \quad-I_{a} R(\lambda-i 0) I_{b} E_{\beta} R(\lambda+i 0) I_{a} \\
& +I_{a} R(\lambda-i 0) E_{\beta} I_{b} R(\lambda+i 0) I_{a},
\end{aligned}
$$

where $K(\lambda)^{*}:=-I_{a}+I_{a} R(\lambda-i 0) I_{b}$. This relation together with (3.21) implies the desired result.

Proof of Theorem 1.1 (ii). Let $a=b$. Then, by Lemma 3.2, we have

$$
\begin{aligned}
\boldsymbol{\sigma}_{\alpha \rightarrow \beta}(\lambda(v), \boldsymbol{\omega})= & \left(2 / v^{2}\right) \operatorname{Im}\left(E_{\beta}(L(v, \omega)-i 0)^{-1} I_{a} \psi_{\alpha}, I_{a} \psi_{\alpha}\right) \\
& +\left(2 / v^{3}\right) \operatorname{Im}\left(E_{\beta} I_{b}(L(v, \boldsymbol{\omega})-i 0)^{-1} I_{a} \psi_{\alpha},(L(v, \boldsymbol{\omega})-i 0)^{-1} I_{a} \psi_{\alpha}\right),
\end{aligned}
$$

where we have used $E_{\beta} \exp \left(i n_{a} v \omega \cdot y\right)=\exp \left(i n_{a} v \omega \cdot y\right) E_{\beta}$, which follows from $a=b$ and the definition of $E_{\beta}$. By Proposition 3.1 (i) with $k=0$ and $(3.19)^{\prime}$ the second term is $O\left(v^{-3}\right)$ uniformly in $\omega \in S^{N-1}$ as $v \rightarrow+\infty$. Next we will show that

$$
\left\|\left((L(v, \omega)-i 0)^{-1}-\left(-i \omega \cdot \nabla_{y}-i 0\right)^{-1}\right) I_{a} \psi_{\alpha}\right\|_{0,-s}=o(1)
$$

uniformly for $\omega \in S^{N-1}$ as $v \rightarrow+\infty$ for $s>1 / 2$. By the resolvent equation we 
have

$$
\begin{aligned}
& (L(v, \omega)-i \varepsilon)^{-1} I_{a} \psi_{a}-\left(-i \omega \cdot \nabla_{y}-i \varepsilon\right)^{-1} I_{a} \psi_{a} \\
& =-v^{-1}(L(v, \omega)-i \varepsilon)^{-1} \cdot\left(H-\lambda_{a}\right)\left(H_{0}-i\right)^{-1} \cdot\left(-i \omega \cdot \nabla_{y}-i \varepsilon\right)^{-1}\left(H_{0}-i\right) I_{a} \psi_{\alpha}
\end{aligned}
$$

for each $\varepsilon>0$, and this yields

$$
\left\|\left((L(v, \omega)-i \varepsilon)^{-1}-\left(-i \omega \cdot \nabla_{y}-i \varepsilon\right)^{-1}\right) I_{a} \psi_{a}\right\| \leqq C(\varepsilon) v^{-1}
$$

for each $\varepsilon>0$. We write

$$
\begin{aligned}
& \left\|\left((L(v, \boldsymbol{\omega})-i 0)^{-1}-\left(-i \omega \cdot \Gamma_{y}-i 0\right)^{-1}\right) I_{a} \psi_{a}\right\|_{0,-s} \\
& \leqq \\
& \quad+\left((L(v, \omega)-i 0)^{-1}-(L(v, \omega)-i \varepsilon)^{-1}\right) I_{a} \psi_{a} \|_{0,-s} \\
& \quad+\left\|\left(\left(-i \omega \cdot \nabla_{y}-i 0\right)^{-1}-\left(-i \omega \cdot \nabla_{y}-i \varepsilon\right)^{-1}\right) I_{a} \psi_{a}\right\|_{0,-s} \\
& \quad+\left\|\left((L(v, \omega)-i \varepsilon)^{-1}-\left(-i \omega \cdot \nabla_{y}-i \varepsilon\right)^{-1}\right) I_{a} \psi_{a}\right\| .
\end{aligned}
$$

Then (3.25) follows from (3.26), (3.10') and Proposition 3.1 (i). Thus by (3.19) and (3.25) the first term of the R. H. S. of (3.24) is

$$
\left(2 / v^{2}\right) \operatorname{Im}\left(E_{\beta}\left(-i \omega \cdot \nabla_{y}-i 0\right)^{-1} I_{a} \psi_{a}, I_{a} \psi_{\alpha}\right)+o\left(v^{-2}\right)
$$

uniformly in $\omega \in S^{N-1}$ as $v \rightarrow+\infty$. Hence,

$$
\sigma_{a \rightarrow \beta}(\lambda(v), \omega)=\left(2 / v^{2}\right) \operatorname{Im}\left(E_{\beta}\left(-i \omega \cdot \nabla_{y}-i 0\right)^{-1} I_{a} \psi_{a}, I_{a} \psi_{a}\right)+o\left(v^{-2}\right),
$$

uniformly in $\omega \in S^{N-1}$ as $v \rightarrow+\infty$. Since

$$
\left(\left(-i \omega \cdot \bar{V}_{y}-i 0\right)^{-1} I_{a} \psi_{\alpha}\right)(x, \eta+t \omega)=i \psi_{a}(x) F(x, \eta+t \omega),
$$

where $\eta \in \Pi_{\omega}, t \in \boldsymbol{R}$ and

$$
F(x, \eta+t \omega)=\int_{-\infty}^{\iota} I_{a}(x, \eta+s \omega) d s
$$

we have

$$
\begin{aligned}
& 2 i \operatorname{Im}\left(E_{\beta}\left(-i \omega \cdot \nabla_{y}-i 0\right)^{-1} I_{a} \psi_{a}, I_{a} \psi_{a}\right) \\
& =i \int_{I_{\omega}} d \eta \int \phi_{\alpha}(x) \overline{\psi_{\beta}(x)} d x \int \overline{\psi_{a}\left(x^{\prime}\right)} \psi_{\beta}\left(x^{\prime}\right) d x^{\prime} \\
& \quad \times \int_{-\infty}^{\infty} \frac{d}{d t}\left(F(x, \eta+t \omega) F\left(x^{\prime}, \eta+t \omega\right)\right) d t \\
& =i \int_{I I_{\omega}}\left|\left(W_{a}(\cdot ; \omega, \eta) \psi_{a}, \phi_{\beta}\right)_{a}\right|^{2} d \eta .
\end{aligned}
$$

This completes the proof.

3.3 .

Proof of Theorem 1.1 (iii). We assume $(\mathrm{V})_{0}$ and $(\mathrm{Z})$. Theorem 2.5 yields 


$$
\begin{aligned}
\sigma_{\alpha}(\lambda(v), \omega) & =-2(2 \pi)^{N-1}\left(n_{a} v\right)^{1-N}\left(\operatorname{Re} T_{\alpha \rightarrow a}(\lambda(v))\right)(\omega, \omega) \\
& =2 v^{-2} \operatorname{Im}\left((L(v, \omega)-i 0)^{-1} I_{a} \psi_{a}, I_{a} \psi_{a}\right) .
\end{aligned}
$$

Thus, by (3.25), we have

$$
\begin{aligned}
\sigma_{\alpha}(\lambda(v), \omega) & =2 v^{-2} \operatorname{Im}\left(\left(-i \omega \cdot V_{y}-i 0\right)^{-1} I_{a} \psi_{a}, I_{a} \psi_{\alpha}\right)+o\left(v^{-2}\right) \\
& =v^{-2} \int_{I_{\omega}}\left\|W_{a}(\cdot ; \omega, \eta) \psi_{a}\right\|_{a}^{2} d \eta+o\left(v^{-2}\right) \quad(\text { see }(3.29))
\end{aligned}
$$

uniformly in $\omega \in S^{N-1}$ as $v \rightarrow+\infty$. This proves (1.21). Under assumptions $(\mathrm{V})_{0}$ and $(Z)$, the set $\left\{\lambda_{\alpha} ; \alpha \in \Gamma_{2}, D(\alpha)=a\right\}$ coincides with the set of all eigenvalues of $h_{a}$. Thus, by (1.15), (3.31), (1.18), (1.19), we obtain

$$
\sigma_{a \rightarrow 0}(\lambda(v), \omega)=v^{-2} \int_{\Pi_{\omega}}\left\|P^{c}\left(h_{a}\right) W_{a}(\cdot ; \omega, \eta) \psi_{a}\right\|_{a}^{2} d \eta+o\left(v^{-2}\right)
$$

uniformly in $\omega \in S^{N-1}$ as $v \rightarrow \infty$. This completes the proof.

\section{4 .}

Proof of Theorem 1.2. We assume $(V)_{3 / 2}$. Then, $I_{a} \dot{\psi}_{\alpha} \in H_{2}^{2}\left(\boldsymbol{R}^{2 N}\right)$ and $(U)_{1}$ is satisfied. Thus, by replacing (3.25) by (3.10) in the above proofs, we get the desired results.

\section{$\S 4$. Abstract Theory for Resolvent Estimates}

In this section we give an abstract theorem for the proof of Proposition 3.1. This theorem is a slight extension of Theorem 2.2 of [JMP] (see also [J], [I]). Throughout this section we work on an abstract Hilbert space $\boldsymbol{H}$ and denote by \|\| the operator norm of bounded operators on $\boldsymbol{H}$.

Definition 4.1. (I) Let $A$ be a self-adjoint operator in $\boldsymbol{H}$ and $d \in \boldsymbol{N}$. We denote by $S_{d}(A)$ the set of all self-adjoint operators $K$ in $\boldsymbol{H}$ satisfying the following properties $(\mathrm{A}-\mathrm{i}) \sim(\mathrm{A}-\mathrm{iv})$.

(A-i) $D(K) \cap D(A)$ is a core for $K$.

(A-ii) $\exp ($ it $A)$ leaves $D(K)$ invariant, and for each $f \in D\left(K^{\prime}\right)$

$$
\sup _{|t| \leqq 1}\|K \exp (i t A) f\|<\infty .
$$

(A-iii) Let $K^{(0)}=K$. There are self-adjoint operators $i K^{(1)}, \cdots, i^{d} K^{(d)}$ satisfying the following:

$$
D\left(i^{j} K^{(j)}\right) \supset D(K) \quad(j=1, \cdots, d),
$$

the form $i\left[i^{j-1} K^{(j-1)}, A\right]$ defined on $D(K) \cap D(A)$ is bounded from below and closable, and the self-adjoint operator associated with its closure is $i^{j} K^{(j)}(j=$ $1, \cdots, d)$. Here, $[$,$] means the commutator: ([B, C] f, g)=\left(C f, B^{*} g\right)-$ 
$\left(B f, C^{*} g\right)$.

(A-iv) The form $\left[K^{(d)}, A\right]$, defined on $D(K) \cap D(A)$, extends to a bounded operator from $\boldsymbol{H}_{+2}$ to $\boldsymbol{H}_{-2}$, which is denoted by $\left[K^{(d)}, A\right]_{0}$, where $\boldsymbol{H}_{+2}$ is the domain $D(K)$ with the graph norm $\|f\|_{+2}:=\|(K+i) f\|$ and $\boldsymbol{H}_{-2}$ is the dual of $\boldsymbol{H}_{+2}$ obtained via the inner product in $\boldsymbol{H}$.

(II) Let $A$ be a self-adjoint operator in $\boldsymbol{H}$ and $d \in N$. We denote by $B_{d}(A)$ the set of all bounded operators $W$ on $\boldsymbol{H}$ satisfying the following property $(\mathrm{A}-\mathrm{V})$.

(A-v) Let $W^{(0)}=W$. There are bounded operators $W^{(1)}, \cdots, W^{(d)}$ on $\boldsymbol{H}$ satisfying the following properties:

The form $\left[W^{(j-1)}, A\right]$, defined on $D(A)$, extends to the bounded operator $W^{(j)}(j=1, \cdots, d)$.

For $K \in S_{d}(A)$ and $W \in B_{d}(A)$, we set

$$
\begin{gathered}
\|K\|_{S_{d}(A)}:=\sum_{j=1}^{d}\left\|K^{(j)}(K+i)^{-1}\right\|+\left\|(K+i)^{-1}[K, A]_{0}(K+i)^{-1}\right\|, \\
\|W\|_{B_{d}(A)}:=\sum_{j=1}^{d}\left\|W^{(j)}\right\| .
\end{gathered}
$$

To state our main results in this section, we prepare some notations. Let $I$ be a compact interval in $R$ and $I_{ \pm}:=\{z \in C ; \operatorname{Re} z \in I, 0< \pm \operatorname{Im} z<1\}$. We fix a smooth function $\chi(t)$ on $\boldsymbol{R}$ such that $0 \leqq \chi \leqq 1, \chi(t)=1$ on $I$ and $\operatorname{supp} \chi(\operatorname{supp}=$ support) is contained in a small neighborhood of $I$.

Theorem 4.2. Let $I, \chi$ be as abov'e, and $A$ a self-adjoint operator in $\boldsymbol{H}$, $d \in \boldsymbol{N}$, and $K_{1}, \cdots, K_{d} \in S_{d}(A)$. Furthermore, if $d \geqq 2$, let $W_{1}, \cdots, W_{d-1} \in B_{d}(A)$. Assume $K_{j}$ satisfies:

$$
\chi\left(K_{j}\right) i K_{j}^{(1)} \chi\left(K_{j}\right) \geqq C_{0} \chi\left(K_{j}\right)^{2}, \quad j=1, \cdots, d,
$$

for some $C_{0}>0$. We define $D(z), z \in \boldsymbol{C} \backslash \boldsymbol{R}$, by

$$
D(z):=\langle A\rangle^{-s}\left(K_{1}-z\right)^{-1}\langle A\rangle^{-s}
$$

for $d=1$,

$$
D(z):=\langle A\rangle^{-s}\left(K_{1}-z\right)^{-1} W_{1}\left(K_{2}-z\right)^{-1} \cdots W_{d-1}\left(K_{d}-z\right)^{-1}\langle A\rangle^{-s}
$$

for $d \geqq 2$, where $s$ is a real with $s\rangle d-(1 / 2)$ and $\langle A\rangle:=\left(1+|A|^{2}\right)^{1 / 2}$. Then, the following (i), (ii) and (iii) hold.

(i)

$$
\sup _{z \in I_{ \pm}}\|D(z)\| \leqq C<\infty
$$

(ii)

$$
\left\|D(z)-D\left(z^{\prime}\right)\right\| \leqq C\left|z-z^{\prime}\right|^{\delta_{0}},
$$


for $z, z^{\prime} \in I_{ \pm}$, where $\delta_{0}=\left(1+(s d /(s-d+1 / 2))^{-1}\right.$. $\lambda \in I$.

(iii) The norm limits $D(\lambda \pm i 0):=\lim _{\varepsilon \downarrow 0} D(\lambda \pm i \varepsilon)$ exist in $\boldsymbol{B}(\boldsymbol{H})$ uniformly in

Moreover, if $A, K_{1}, \cdots, K_{d}$ (and $W_{1}, \cdots, W_{d-1}$ if $d \geqq 2$ ) depend on a parameter $\nu$ such that $I, \chi$ and $C_{0}$ can be taken independently of $\nu$ and that $\left\|K_{j}\right\|_{S_{d}(A)}$ $(j=1, \cdots, d)$ (and $\left\|W_{j}\right\|_{B_{d}(A)}(j=1, \cdots, d-1)$ if $\left.d \geqq 2\right)$ remain bounded in $\nu$, then $C$ can be taken independently of $\nu$.

For $0<|\varepsilon| \ll 1$ the operator

$$
Q_{j}(\varepsilon):=\sum_{m=1}^{d} \frac{\varepsilon^{m}}{m !} K_{j}^{(m)}, \quad j=1, \cdots, d,
$$

is $K_{j}$-bounded with $K_{j}$-bound $<1$ by (A-iii). Thus $K_{j}+Q_{\jmath}(\varepsilon)$ is a closed operator with $D\left(K_{j}+Q_{j}(\varepsilon)\right)=D\left(K_{j}\right)$.

Lemma 4.3 ([JMP], Lemma 3.1). There exists a $\varepsilon_{1}>0$ such that the following properties hold for $0< \pm \varepsilon<\varepsilon_{1}, z \in I_{ \pm}, j=1, \cdots, d$ :

(i) $K_{j}+Q_{j}(\varepsilon)-z$ has a bounded inverse $G_{j, z}(\varepsilon) \in \boldsymbol{B}(\boldsymbol{H})$

(ii) $G_{j, z}(\varepsilon)$ satisfies the following estimates:

$$
\begin{gathered}
\left\|G_{j, z}(\varepsilon)\right\| \leqq C|\varepsilon|^{-1}, \\
\left\|\left(K_{\jmath}+i\right) G_{j, z}(\varepsilon)\right\|+\left\|G_{j, z}(\varepsilon)\left(K_{\jmath}+i\right)\right\| \leqq C|\varepsilon|^{-1}, \\
\left\|\left(K_{j}+i\right) G_{j, z}(\varepsilon)\langle A\rangle^{-1}\right\|+\left\|\langle A\rangle^{-1} G_{\jmath, z}(\varepsilon)\left(K_{\jmath}+i\right)\right\| \leqq C|\varepsilon|^{-1 / 2},
\end{gathered}
$$

where $C$ is independent of $\pm \varepsilon \in\left(0, \varepsilon_{1}\right), z \in I_{ \pm}, j=1, \cdots, d$.

(iii) The form $\left[A, G_{j, z}(\varepsilon)\right]$, defined on $D(A)$, extends to a bounded operator $\left[A, G_{j, z}(\varepsilon)\right]_{0}$ on $\boldsymbol{H} . \quad G_{j, z}(\varepsilon)$ maps $D(A)$ into $D(A) \cap D\left(K_{\jmath}\right)$.

(iv) For each $z \in I_{+}\left(\right.$resp. $\left.I_{-}\right), G_{\jmath_{3}}(\varepsilon) \in C^{1}\left(\left(0, \varepsilon_{1}\right) ; \boldsymbol{B}(\boldsymbol{H})\right)\left(\right.$ resp. $C^{1}\left(\left(-\varepsilon_{1}, 0\right)\right.$; $\boldsymbol{B}(\boldsymbol{H}))$ and

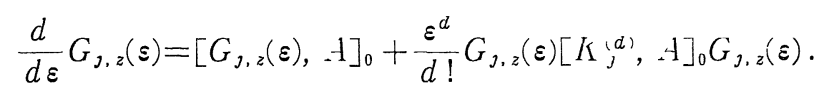

Moreover, if $A, K_{1}, \cdots, K_{d}$ depends on a parameter $\nu$ such that $I, \chi$ and $C_{0}$ can be taken independently of 2 and that $\left\|K_{j}\right\|_{S_{d}(A)}(j=1, \cdots, d)$ remain bounded in $\nu$, then $\varepsilon_{1}, C$ can be taken independently of $\nu$.

For properties (i) (iv) of the lemma it suffices to prove them for each $j$. For the proof, see [JMP]. The last part can be shown by carefully checking the estimates carried out in [JMP] (see also [M], [PSS]).

Lemma 4.4 ([I], Lemma 3.5). Let $f_{k}(\varepsilon)=|\log \varepsilon|$ for $k=0$ and $f_{k}(\varepsilon)=\varepsilon^{-k}$ for $k \in \boldsymbol{N}$. Assume that a $\boldsymbol{B}(\boldsymbol{H})$-valued $C^{1}$-function $X(\varepsilon), 0<\varepsilon<\varepsilon_{1} \quad\left(\varepsilon_{1}>0\right)$, satisfies: 


$$
\begin{gathered}
\|(d / d \varepsilon) X(\varepsilon)\| \leqq C_{1}\left(\|X(\varepsilon)\|^{p} \cdot \varepsilon^{-q}+f_{k}(\varepsilon)+1\right), \\
\|X(\varepsilon)\| \leqq C_{2} \varepsilon^{-r},
\end{gathered}
$$

where $p, q, r, C_{1}, C_{2}$ are constants satisfying $0 \leqq p<1,0 \leqq q<1, r \geqq 0, C_{1}, C_{2}>0$. Then $X(\varepsilon)$ satisfies the following estimates:

$$
\begin{array}{ll}
\|X(\varepsilon)\|=C \cdot \varepsilon^{-k+1} & \text { when } k \geqq 2, \\
\|X(\varepsilon)\| \leqq C|\log \varepsilon| & \text { when } k=1, \\
\|X(\varepsilon)\| \leqq C & \text { when } k=0,
\end{array}
$$

where $C=C\left(C_{1}, C_{2}, \varepsilon_{1}, p, q, r\right)>0$. Furthermore, when $k=0$, the norm limit $X(0):=\lim _{\varepsilon \downarrow 0} X(\varepsilon)$ exists in $\boldsymbol{B}(\boldsymbol{H})$.

For the proof, see [I].

For the proof of Theorem 4.2 for the case $d=1$, see $[\mathrm{M}]$, [PSS]. We can also prove this theorem for $d \geqq 2$ in the same way as in Theorem 3.3 in [I]. The proof is a slight modification of the proof of Theorem 2.2 in [JMP]. But, for the sake of completeness, we give the proof of Theorem 4.2 for $d \geqq 2$.

Proof of Theorem 4.2. We give only the proof for the case $z \in I_{+}$.

(i) For multi-indices of nonnegative integers $\alpha=\left(\alpha_{1}, \cdots, \alpha_{t-1}\right), \beta=\left(\beta_{1}, \cdots\right.$, $\left.\beta_{d-1}\right)$ we write $\left|\alpha^{\prime}\right|=\alpha_{1}+\cdots+\alpha_{d-1}$, and $\alpha \leqq \beta$ if and only if $\alpha, \leqq \beta_{\jmath}$ for all $i$. Let $M_{a}$ be a family of all multi-indices $\beta$ with $\alpha \leqq \beta,|\beta|=|\alpha|+1$. Namely $\beta \in M_{a}$ implies that $\alpha_{j}=\beta_{j}-1$ for some $j$ and $\beta_{\imath}=\alpha_{\imath}$ for $i \mp j$. We set

$$
F_{z}^{a}(\varepsilon):=\langle A\rangle^{-s} G_{1, z}(\varepsilon) W_{1}^{\left(a_{1}\right)} G_{2}(\varepsilon) \cdots W_{d-1}^{(o d-1)} G_{d, \varepsilon}(\varepsilon)\langle A\rangle^{-s}
$$

for $z \in I_{+}, \quad \varepsilon>0, \alpha=\left(\alpha_{1}, \cdots, \alpha_{d-1}\right)$ with $|\alpha| \leqq d$.

By Lemma 4.3 (iv), we have for $|\alpha| \leqq d-1$.

$$
\begin{aligned}
\frac{d}{d \varepsilon} F_{z}^{\alpha}(\varepsilon)= & \langle A\rangle^{-s}\left(\frac{d}{d \varepsilon} G_{1, z}(\varepsilon)\right) W_{1}^{\left(a_{1}\right)} G_{2}(\varepsilon) \cdots W_{d-1}^{\left(a_{d-1}\right)} G_{d, z}(\varepsilon)\langle A\rangle^{-s} \\
& +\cdots+\langle A\rangle^{-s} G_{1, z}(\varepsilon) W_{1}^{\left(a_{1}\right)} G_{2, z}(\varepsilon) \cdots W_{d-1}^{\left(\alpha_{d-1}\right)}\left(\frac{d}{d \varepsilon} G_{d, z}(\varepsilon)\right)\langle A\rangle^{-s} \\
= & \langle A\rangle^{-s}\left\{\left[G_{1, z}(\varepsilon), A\right]_{0} W_{1}^{\left(n_{1}\right)} G_{2, z}(\varepsilon) \cdots W_{d-1}^{\left(\alpha_{d-1}\right)} G_{d, z}(\varepsilon)\right. \\
& \left.\left.+\cdots+G_{1, z}(\varepsilon) W_{1}^{\left(a_{1}\right)} G_{2, z}(\varepsilon) \cdots W_{d-1}^{\left(\alpha_{d-1}\right)}\left[G_{d, z}(\varepsilon), A\right]\right]_{0}\right\}\langle A\rangle^{-s} \\
& +\frac{\varepsilon^{d}}{d !}\langle A\rangle^{-s}\left\{G_{1, z}(\varepsilon)\left[K_{1}^{(d)}, A\right]_{0} G_{1, z}(\varepsilon) \cdots W_{d-1}^{\left(a_{d-1}\right)} G_{d, z}(\varepsilon)\right. \\
& \left.+\cdots+G_{1, z}(\varepsilon) W_{1}^{\left(a_{1}\right)} \cdots W_{d-1}^{\left(\alpha_{d-1}\right)} G_{d, z}(\varepsilon)\left[K_{d}^{(d)}, A\right]_{0} G_{d, z}(\varepsilon)\right\}\langle A\rangle^{-s} \\
= & I_{1}(\varepsilon)+I_{2}(\varepsilon) .
\end{aligned}
$$


First we estimate $I_{2}(\varepsilon)$. Since $s>1$ and $W_{j}^{\left(a_{j}\right)}(j=1, \cdots, d-1),\left(K_{n}+i\right)^{-1}\left[K_{n}^{(d)}\right.$, $A]_{0}\left(K_{n}+i\right)^{-1}(n=1, \cdots, d)$ are bounded by $(\mathrm{A}-\mathrm{v}),(\mathrm{A}-\mathrm{iv})$, we have

$$
\left\|I_{2}(\varepsilon)\right\| \leqq C \cdot \varepsilon^{d} \cdot \varepsilon^{-1 / 2} \cdot \varepsilon^{-d+1} \cdot \varepsilon^{-1 / 2} \leqq C
$$

by Lemma 4.3 (ii).

Next we estimate $I_{1}(\varepsilon)$. Noting that $G_{n, z}(\varepsilon)$ maps $D(A)$ into $D(A)$ and $W_{j}^{(\alpha)}$ maps $D(A)$ into $D(A)$, as follows from (A-v) and Lemma 4.3 (iii), we have, by elementary computation,

$$
I_{1}(\varepsilon)=\left[F_{z}^{\alpha}(\varepsilon), 4\right]-\sum_{\beta \in M_{a}} F_{z}^{\beta}(\varepsilon) .
$$

Since $\left\|\langle A\rangle^{s} F_{z}^{\alpha}(\varepsilon)\right\|,\left\|F_{z}^{\alpha}(\varepsilon)\langle A\rangle^{s}\right\| \leqq C \cdot \varepsilon^{-d+(1 / 2)}$ by Lemma 4.3 (ii) and

$$
\begin{gathered}
\left\|F_{z}^{\alpha}(\varepsilon)\langle A\rangle\right\| \leqq\left\|F_{z}^{\alpha}(\varepsilon)\right\|^{1-(1 / s)}\left\|F_{z}^{a}(\varepsilon)\langle A\rangle^{s}\right\|^{1 / s}, \\
\left\|\langle A\rangle F_{z}^{\alpha}(\varepsilon)\right\| \leqq\left\|F_{z}^{\alpha}(\varepsilon)\right\|^{1-(1 / s)}\left\|\langle A\rangle^{s} F_{z}^{\alpha}(\varepsilon)\right\|^{1 / s}
\end{gathered}
$$

by interpolation, we have

$$
\begin{aligned}
\left\|\left[F_{z}^{\alpha}(\varepsilon), A\right]\right\| & \leqq F_{z}^{\alpha}(\varepsilon)\langle A\rangle\|+\|\langle A\rangle F_{z}^{a}(\varepsilon) \| \\
& \leqq C \cdot\left\|F_{z}^{\alpha}(\varepsilon)\right\|^{1-(1 / s)} \varepsilon^{(-d+(1 / 2)) / s} .
\end{aligned}
$$

Thus we get

$$
\left\|I_{1}(\varepsilon)\right\| \leqq C\left(\left\|F_{z}^{a}(\varepsilon)\right\|^{1-(1 / s)} \varepsilon^{(-d+(1 / 2)) / s}+\sum_{\beta \in M_{a}}\left\|F_{z}^{\beta}(\varepsilon)\right\|\right) .
$$

Therefore $F_{z}^{\alpha}(\varepsilon)$ satisfies by (4.16), (4.17) and (4.19)

$$
\left\|\frac{d}{d \varepsilon} F_{z}^{\alpha}(\varepsilon)\right\| \leqq C\left(\left\|F_{z}^{\alpha(}(\varepsilon)\right\|^{1-(1 / s)} \varepsilon^{-m}+\sum_{\beta \in M_{\alpha}}\left\|F_{z}^{\beta}(\varepsilon)\right\|+1\right)
$$

for all multi-indices $\alpha$ with $|\alpha| \leqq d-1$ where $m=(d-(1 / 2)) / s$.

Furthermore, it follows from Lemma 4.3 (ii) that

$$
\left\|F_{z}^{\gamma}(\varepsilon)\right\| \leqq C \varepsilon^{-d+1}
$$

for all multi-indices $\gamma$ with $|\gamma| \leqq d$.

Let $|\alpha|=d-1$. Then we have by (4.20) and (4.21)

$$
\left\|\frac{d}{d \varepsilon} F_{z}^{\alpha}(\varepsilon)\right\| \leqq C\left(\left\|F_{z}^{\alpha}(\varepsilon)\right\|^{1-(1 / s)} \varepsilon^{-m}+\varepsilon^{-d+1}+1\right) .
$$

Applying Lemma $4.4(p=1-(1 / s), q=m ; p, q \in[0,1)$ by $s>d-(1 / 2))$, we have

$$
\left\|F_{z}^{\alpha}(\varepsilon)\right\| \leqq C \varepsilon^{-d+2} .
$$

Next let $|\alpha|=d-2$. Then $|\beta|=d-1$ for $\beta \in M_{\alpha}$. Thus we obtain by

$$
\left\|\frac{d}{d \varepsilon} F_{z}^{\alpha}(\varepsilon)\right\| \leqq C\left(\left\|F_{z}^{\alpha}(\varepsilon)\right\|^{1-(1 / s)} \varepsilon^{-m}+\varepsilon^{-d+2}+1\right)
$$


Applying Lemma 4.4, we have

$$
\left\|F_{z}^{\alpha}(\varepsilon)\right\| \leqq C \varepsilon^{-d+3} .
$$

Continuing, we have for $|\alpha|=0$

$$
\left\|\frac{d}{d \varepsilon} F_{z}^{\alpha}(\varepsilon)\right\| \leqq C\left(\left\|F_{z}^{\alpha}(\varepsilon)\right\|^{1-(1 / s)} \varepsilon^{-n}+|\log \varepsilon|+1\right) .
$$

Thus we have the following estimate, by Lemma 4.4,

$$
\sup _{z \in I_{T}, 0<\varepsilon<1}\left\|F_{z}(\varepsilon)\right\| \leqq C<\infty,
$$

where $F_{z}(\varepsilon):=F_{z}^{a}(\varepsilon)$ for $|\alpha|=0$.

We set $R_{n}(z)=\left(K_{n}-z\right)^{-1}$. Since $\lim _{\varepsilon \downarrow 0}\left\|Q_{n}(\varepsilon) R_{n}(z)\right\|=0$ for each $z \in \boldsymbol{C} \backslash \boldsymbol{R}$ by (A-iii), $1+Q_{n}(\varepsilon) R_{n}(z)$ has a bounded inverse, and so

$$
G_{n, z}(\varepsilon)=R_{n}(z)\left(1+Q_{n}(\varepsilon) R_{n}(z)\right)^{-1}
$$

holds for each $z \in \boldsymbol{C} \backslash \boldsymbol{R}$ when $\varepsilon>0$ is small. Therefore we get

$$
\lim _{\varepsilon \downarrow 0} G_{n, z}(\varepsilon)=R_{n}(z) \quad(n=1, \cdots, d)
$$

in the norm of $\boldsymbol{B}(\boldsymbol{H})$ for each $z \in \boldsymbol{C} \backslash \boldsymbol{R}$, and so we have by (4.24)

$$
\sup _{z \in I_{+}}\|D(z)\| \leqq C .
$$

(ii) By (4.23), (4.24) we obtain

$$
\left\|\frac{d}{d \varepsilon} F_{z}(\varepsilon)\right\| \leqq C\left(\varepsilon^{-m}+1\right) .
$$

Integrating this, we have, by noting $0<m<1$,

$$
\left\|F_{z}(\varepsilon)-F_{z}(0)\right\| \leqq C \cdot \varepsilon^{1-m} .
$$

On the other hand $G_{n, z}(\varepsilon)$ is differentiable in $z \in I_{+}$for each $\varepsilon>0$ by Lemma 4.3. We have the following estimate by Lemma 4.3 (ii):

which implies

$$
\begin{aligned}
\left\|\frac{d}{d z} F_{z}(\varepsilon)\right\| \leqq & \left\|\langle A\rangle^{-s} G_{1, z}(\varepsilon)^{2} W_{1} \cdots G_{d, z}(\varepsilon)\langle A\rangle^{-s}\right\| \\
& +\cdots+\left\|\langle A\rangle^{-s} G_{1, z}(\varepsilon) W_{1} \cdots W_{d-1} G_{d, z}(\varepsilon)^{2}\langle A\rangle^{-s}\right\| \\
\leqq & C \cdot \varepsilon^{-d},
\end{aligned}
$$

$$
\left\|F_{z}(\varepsilon)-F_{z^{\prime}}(\varepsilon)\right\| \leqq C \cdot \varepsilon^{-d}\left|z-z^{\prime}\right|
$$

for $z, z^{\prime} \in I_{+}, \varepsilon>0$. Let $\varepsilon=\left|z-z^{\prime}\right|^{\delta_{1}}, \delta_{1}=(1-m)^{-1} \delta_{0}$ (see (4.5) for $\delta_{0}$ ). Then by (4.25), (4.26) we have 


$$
\begin{aligned}
\left\|F_{z}(0)-F_{z^{\prime}}(0)\right\| & \leqq\left\|F_{z}(0)-F_{z}(\varepsilon)\right\|+\left\|F_{z}(\varepsilon)-F_{z^{\prime}}(\varepsilon)\right\|+\left\|F_{z^{\prime}}(\varepsilon)-F_{z^{\prime}}(0)\right\| \\
& \leqq C \cdot\left|z-z^{\prime}\right|^{\delta_{0}} .
\end{aligned}
$$

Thus we have proved (ii). (iii) follows from (ii).

The uniformity of the choice of $C$ can be obtained if one takes into consideration the last part of Lemma 4.3 and the proof carried out above.

\section{§. Proof of Proposition 3.1}

In this section we will prove Proposition 3.1 by applying Theorem 4.2. Throughout this section we assume $(\mathrm{U})$, and fix an integer $k$ with $0 \leqq k \leqq \ell$. Furthermore we define a set $\Omega:=\left\{(v, \omega) ; v>v_{0}, \omega \in S^{v-1}\right\}$ for $v_{0}>0$. Let $A_{0}$ be the generator of dilations on $\boldsymbol{R}^{2 \Lambda}$ :

$$
A_{0}=(1 / 2 i)\left(x \cdot F_{x}+V_{x} \cdot x+y \cdot \Gamma_{y}+V_{y} \cdot y\right),
$$

which is self-adjoint in $\mathscr{H}$ with a core $\mathcal{S}=\mathcal{S}\left(\boldsymbol{R}^{2 v}\right)$, the Schwartz space of rapidly decreasing functions. Thus, the operator $A(\tau), \tau=(v, \omega) \in \Omega$, defined by

$$
\begin{aligned}
A(\tau): & =\left(n_{a} v\right)^{-1} A_{0}+\omega \cdot y \\
& =\left(n_{a} v\right)^{-1} \exp \left(-i n_{a} v \omega \cdot y\right) A_{0} \exp \left(i n_{a} v \omega \cdot y\right),
\end{aligned}
$$

is self-adjoint in $\mathscr{H}$ with a core $\mathcal{S}$. For notational brevity we write $L(\tau)=$ $L(v, \boldsymbol{\omega})$ for $\tau=(v, \boldsymbol{\omega}) \in \Omega$. Then, a simple calculation yields

$$
i[L(\tau), A(\tau)]=2\left(n_{a} v\right)^{-1} L(\tau)+\left(n_{a} v^{2}\right)^{-1}\left(2\left[V, A_{0}\right]-2 V+2 \lambda_{a}\right)+1
$$

on $\mathcal{S}$, where $i\left[V, A_{0}\right]$ is an operator of multiplication:

$$
i\left[V, A_{0}\right]=-\sum_{1 \leqq i<j \leqq 3} V_{i j}^{(1)}\left(r_{2}-r_{\jmath}\right),
$$

where

$$
V_{i j}^{(n)}(x)=\left(x \cdot \Gamma_{x}\right)^{n} V_{i j}(x), \quad n \in \boldsymbol{N} .
$$

Lemma 5.1. (i) $L(\tau) \in S_{\iota+1}(A(\tau))$ for each $\tau \in \Omega$ (see Definition 4.1) and

$$
\sup _{\tau \in \Omega}\|L(\tau)\|_{S_{\ell+1}(A(\tau))}<\infty .
$$

(ii) Fix a smooth function $\chi(t)$ on $\boldsymbol{R}$ with $\chi=1$ on $[-1 / 2,1 / 2]$ and $\operatorname{supp} \chi \subset$ $[-1,1]$. Then,

$$
\chi(L(\tau)) i[L(\tau), A(\tau)] \chi(L(\tau)) \geqq(1 / 2) \chi(L(\tau))^{2}
$$

for all $\tau \in \Omega$ if $\eta_{0} \gg 1$.

Proof. (i) Since $\mathcal{S}$ is a common core for $L(\tau)$ and $A(\tau)$, (A-i) follows. (A-ii) can be easily verified by (5.2). By (5.3), (5.4), (5.5) and (U), we see that 
the $n$-th $(0 \leqq n \leqq \ell+2)$ commutator

$$
i^{n}[[\cdots[L(\tau), A(\tau)], \cdots], \Lambda(\tau)]
$$

on $\mathcal{S}$ can be uniquely extended to a self-adjoint operator $i^{n} L^{(n)}(\tau)$ with domain $H^{2}\left(\boldsymbol{R}^{2 v}\right)$. Thus, taking account of the fact that $\mathcal{S}$ is a common core for $L(\tau)$ and $A(\tau)$, we can verify (A-iii), (A-iv). Therefore, we see that $L(\tau) \in S_{\iota+1}(A(\tau))$ for each $\tau \in \Omega$. (5.6) can be verified by using (5.3).

(ii) For $v_{0} \gg 1$, we get, by (5.3),

$$
\begin{aligned}
& \chi(L(\tau)) i[L(\tau), H(\tau)] \chi(L(\tau)) \\
& \quad \geqq\left(-\left(2 / n_{a} v\right)-\left(1 / n_{a} v^{2}\right)\left\|i\left[V, 1_{0}\right]-2 V+2 \lambda_{a}\right\|+1\right) \chi(L(\tau))^{2} \\
& \quad \geqq(1 / 2) \chi(L(\tau))^{2} .
\end{aligned}
$$

This completes the proof.

For $\tau \in \Omega$ and $z \in \boldsymbol{C} \backslash \boldsymbol{R}$, we write $R(\tau, z)=(L(\tau)-z)^{-1}$. It is not difficult to check, by using $V R(\tau, i)=R(\tau, i) \bar{V}-R(\tau, i) v^{-1}(\nabla V) R(\tau, i)$ and $(\mathrm{U})_{\iota}$, the following estimate :

$$
\left.\sup _{\tau \in \Omega}\|R(\tau, i)\|_{\boldsymbol{B}(I I}{ }^{m}, I^{m}\right)<\infty
$$

for $0 \leqq m \leqq 2 \ell+2$, where $H^{m}=H_{0}^{m}\left(\boldsymbol{R}^{2 N}\right)$.

Lemma 5.2. Let $m$ be an integer with $0 \leqq m \leqq 2 \ell+2$. Then

$$
\sup _{\tau \in \Omega} i^{-1}\|R(\tau, i)\|_{\boldsymbol{B}\left(H^{m}, H^{m+1}\right)<\infty} .
$$

Proof. Let $L_{0}(\tau):=v^{-1}\left(H_{0}-\lambda_{n}\right)-i \omega \cdot \nabla_{\eta}$ for $\tau=(v, \omega) \subseteq \Omega$. Then, by (5.9), $(U)$, and the resolvent equation

$$
R(\tau, i)=\left(L_{0}(\tau)-i\right)^{-1}-v^{-1}\left(L_{0}(\tau)-i\right)^{-1} V R(\tau, i),
$$

it suffices to show that

$$
\sup _{\tau=(v, \omega) \in \Omega} v^{-1}\left\|\left(L_{0}(\tau)-i\right)^{-1}\right\|_{\boldsymbol{B}\left(H^{m}, H\right.}{ }^{m+1},<\infty,
$$

which is, by the Fourier transform, reduced to the following estimate:

$$
\begin{aligned}
\sup _{\substack{\tau=(\boldsymbol{v}, \boldsymbol{\omega}) \in \Omega \\
\xi, \eta \in \boldsymbol{R}^{N}}} \mid v^{-1}(|\xi|+|\eta|+1) \\
\quad \times\left(\left|v^{-1}\left(\left(1 / 2 m_{a}\right) \xi^{2}+\left(1 / 2 n_{a}\right) \eta^{2}-\lambda_{n}\right)+\omega \cdot \eta\right|+1\right)^{-1} \mid<\infty .
\end{aligned}
$$

Taking account of $\lambda_{a}<0$ and $2 a b \leqq a^{2}+b^{2}$ for $a, b \in \boldsymbol{R}$, we have

$$
|\xi|+|\eta|+1 \leqq v^{-1}\left(\left(1 / 2 m_{a}\right) \xi^{2}+\left(1 / 2 n_{a}\right) \eta^{2}-\lambda_{a}\right)+\omega \cdot \eta+\left(\left(m_{a} / 2\right)+2 n_{a}\right) v+1,
$$

which yields (5.12). This completes the proof. 
Proof of Proposition 3.1 (i).

Case $k=0$. We first consider the case $k=0$. For the proof, it sufficies to show that

$$
\begin{gathered}
\sup _{\tau \in \Omega, 0<\varepsilon<1}\left\|X_{s} R(\tau, i \varepsilon) X_{s}\right\|<\infty, \\
\lim _{\varepsilon, \varepsilon^{\prime} \downarrow 0} \sup _{\tau \in \Omega}\left\|X_{s}\left[R(\tau, i \varepsilon)-R\left(\tau, i \varepsilon^{\prime}\right)\right] X_{s}\right\|=0,
\end{gathered}
$$

where $X_{s}:=\left(1+|x|^{2}+|y|^{2}\right)^{-s / 2}$ and $1 / 2<s \leqq 1$. By the resolvent equation we have

$$
R(\tau, i \varepsilon)=R(\tau, i)+(i \varepsilon-i) R(\tau, i)^{2}+(i \varepsilon-i)^{2} R(\tau, i) R(\tau, i \varepsilon) R(\tau, i) .
$$

Thus, we have only to prove the following estimates:

$$
\begin{gathered}
\sup _{\tau \in \Omega, 0<\varepsilon<1}\left\|\langle A(\tau)\rangle^{-s} R(\tau, i \varepsilon)\langle A(\tau)\rangle^{-s}\right\|\langle\infty, \\
\lim _{\varepsilon, \varepsilon^{\prime} \downarrow 0} \sup _{\tau \in \Omega}\left\|\langle A(\tau)\rangle^{-s}\left[R(\tau, i \varepsilon)-R\left(\tau, i \varepsilon^{\prime}\right)\right]\langle A(\tau)\rangle^{-s}\right\|=0, \\
\sup _{\tau \in \Omega}\left\|X_{s} R(\tau, \pm i)\langle A(\tau)\rangle^{s}\right\|<\infty .
\end{gathered}
$$

(5.16) and (5.17) follow from Theorem 4.2 and Lemma 5.1. By interpolation, it suffices to prove (5.18) for $s=1$. We have

$$
X_{1} R(\tau, \pm i) A(\tau)=X_{1} A(\tau) R(\tau, \pm i)-X_{1} R(\tau, \pm i)[L(\tau), A(\tau)] R(\tau, \pm i)
$$

on $\mathcal{S}$. By (5.3) the operator norm of the second term in the R.H.S. is uniformly bounded in $\tau \in \Omega$, and the norm of the first term is also uniformly bounded in $\tau \in \Omega$ by Lemma 5.2 with $m=0$ because

$$
A(\tau)=\left(n_{a} v i\right)^{-1}\left(x \cdot \nabla_{x}+y \cdot \nabla_{y}+N\right)+\omega \cdot y, \quad \tau=(v, \omega) .
$$

Thus we have proved Proposition 3.1 (i) for $k=0$.

Case $k \geqq 1$. We next consider the case $1 \leqq k \leqq \ell$. We may assume $k+\cdot(1 / 2)$ $<s \leqq k+1$. For the proof, it suffices to prove

$$
\sup _{\tau \in \Omega, 0<\varepsilon<1}\left\|X_{s} D^{r} R(\tau, i \varepsilon)\langle D\rangle^{-k} X_{s}\right\|<\infty,
$$

and

$$
\lim _{\varepsilon, \varepsilon^{\prime} \downarrow 0} \sup _{\tau \in \Omega}\left\|X_{s} D^{r}\left[R(\tau, i \varepsilon)-R\left(\tau, i \varepsilon^{\prime}\right)\right]\langle D\rangle^{-k} X_{s}\right\|=0,
$$

where $|\gamma| \leqq k$ and $D:=\left(\partial_{x}, \partial_{y}\right),\langle D\rangle:=(-\Delta+1)^{1 / 2}$. Taking account of (5.13), (5.14) and

$$
X_{-s} D^{r}\langle D\rangle^{-k} X_{s} \in \boldsymbol{B}(\mathscr{H}) \quad \text { for }|\gamma| \leqq k,
$$

and using 


$$
D R(\tau, i \varepsilon)=R(\tau, i \varepsilon) D-R(\tau, i \varepsilon) v^{-1}(D V) R(\tau, i \varepsilon)
$$

repeatedly, we see that the proof of (5.21) and (5.22) are reduced to the proof of the following:

$$
\begin{gathered}
\sup _{\tau \in \Omega, 0<\varepsilon<1}\left\|X_{s} R(\tau, i \varepsilon)\left(D^{\gamma_{1}} V\right) R(\tau, i \varepsilon) \cdots\left(D^{\gamma m} V\right) R(\tau, i \varepsilon) X_{s}\right\|<\infty, \\
\lim _{\varepsilon, \varepsilon^{\prime} \downarrow 0} \sup _{\tau \in \Omega} \| X_{s}\left\{R(\tau, i \varepsilon)\left(D^{\gamma_{1}} V\right) R(\tau, i \varepsilon) \cdots\left(D^{\gamma_{m}} V\right) R(\tau, i \varepsilon)\right. \\
\left.\quad-R\left(\tau, i \varepsilon^{\prime}\right)\left(D^{\gamma_{1}} V\right) R\left(\tau, i \varepsilon^{\prime}\right) \cdots\left(D^{\gamma m} V\right) R\left(\tau, i \varepsilon^{\prime}\right)\right\} X_{s} \|=0,
\end{gathered}
$$

where $1 \leqq m \leqq k$ and $\sum_{j=1}^{m}\left|\gamma_{j}\right| \leqq k$. Using (5.15) repeatedly, we have

$$
\begin{aligned}
R(\tau, i \varepsilon)= & \sum_{0 \leqq m \leqq 2 k+1}(i \varepsilon-i)^{m} R(\tau, i)^{m+1} \\
& +(i \varepsilon-i)^{2 k+2} R(\tau, i)^{k+1} R(\tau, i \varepsilon) R(\tau, i)^{k+1} .
\end{aligned}
$$

Thus, replacing $R(\tau, i \varepsilon)$ in (5.24), (5.25) with the R. H.S. of (5.26) and taking account of $\|R(\tau, i)\| \leqq 1$, we can reduce the proof of (5.21), (5.22) to that of the following :

$$
\begin{aligned}
& \sup _{\tau \in \Omega, 0<\varepsilon<1} \| X_{s} U_{1} R(\tau, i \varepsilon) U_{2} \cdots R(\tau, i \varepsilon) U_{m+1} X_{s} \|<\infty, \\
& \lim _{\varepsilon, \varepsilon^{\prime} \downarrow 0} \sup _{\tau \in \Omega} \| X_{s} U_{1}\left\{R(\tau, i \varepsilon) U_{2} \cdots R(\tau, i \varepsilon)\right. \\
&\left.\quad-R\left(\tau, i \varepsilon^{\prime}\right) U_{2} \cdots R\left(\tau, i \varepsilon^{\prime}\right)\right\} U_{m+1} X_{s} \|=0 .
\end{aligned}
$$

where $1 \leqq m \leqq k+1$, and each $U_{j}=U_{j}(\tau)$ has the form:

$$
U_{j}=R(\tau, i)^{k+1} Y_{1} \cdots Y_{q} \text { or } Y_{1} \cdots Y_{\mathrm{q}} R(\tau, i)^{k+1},
$$

where $Y_{j}=R(\tau, i)$ or $D^{r} V$ for $|\gamma| \leqq k$.

Consequently, taking account of (5.16), (5.17) in the case $m=1$ it suffices to prove the following:

$$
\begin{gathered}
\sup _{\tau \in \Omega}\left\{\left\|X_{s} U_{1}\langle A(\tau)\rangle^{s}\right\|+\left\|\langle A(\tau)\rangle^{s} U_{m+1} X_{s}\right\|<\infty,\right. \\
\sup _{\tau \in \Omega, 0<\varepsilon<1}\left\|\langle A(\tau)\rangle^{-s} R(\tau, i \varepsilon) U_{2} R(\tau, i \varepsilon) \cdots U_{m} R(\tau, i \varepsilon)\langle A(\tau)\rangle^{-s}\right\|<\infty \quad(m \geqq 2), \\
\lim _{\varepsilon, \varepsilon^{\prime} \downarrow 0} \sup _{\tau \in \Omega} \|\langle A(\tau)\rangle^{-s}\left\{R(\tau, i \varepsilon) U_{2} R(\tau, i \varepsilon) \cdots U_{m} R(\tau, i \varepsilon)\right. \\
\left.-R\left(\tau, i \varepsilon^{\prime}\right) U_{2} R\left(\tau, i \varepsilon^{\prime}\right) \cdots U_{m} R\left(\tau, i \varepsilon^{\prime}\right)\right\}\langle A(\tau)\rangle^{-s} \|=0 \quad(m \geqq 2) .
\end{gathered}
$$

By $(\mathrm{U})_{\iota}$ and (5.3), we can easily see that $U_{j}(\tau) \in B_{\iota+1}(A(\tau))$ and

$$
\sup _{\tau \in \Omega}\left\|U_{\jmath}(\tau)\right\|_{B_{\ell+1}(A(\tau))}<\infty
$$

for each $j$. Hence, (5.31), (5.32) follow from Lemma 5.1 and Theorem 4.2 with $d=m, K_{j}(\tau)=L(\tau)(1 \leqq j \leqq m)$ and $W_{j}(\tau)=U_{j_{+1}}(\tau)(1 \leqq j \leqq m-1)$. 
Finally we prove that $\left\|X_{s} U_{1}\langle A(\tau)\rangle^{s}\right\|, \tau \in \Omega$, is uniformly bounded. The uniform boundedness of $\left\|\langle A(\tau)\rangle^{s} U_{m+1} X_{s}\right\|$ can be proved similarly. We write $U_{1} A(\tau)^{k+1}=A(\tau)^{k+1} U_{1}+\left[L_{1}, A(\tau)^{k+1}\right]$ on $\mathcal{S} . A(\tau)^{k+1}$ is written as

$$
\left.\sum_{\substack{\left|\gamma_{1}\\\right| \gamma_{3} \mid=k i \leq k+1}} C\left(\tau ; \gamma_{1}, \gamma_{2}, \gamma_{3}\right) x^{\gamma_{1}} y^{i_{2}\left(\gamma^{2}-1\right.} D\right)^{\gamma_{3}}
$$

for $\tau=(v, \omega)$, where the constants $C\left(\tau ; \gamma_{1}, \gamma_{2}, \gamma_{3}\right)$ are uniformly bounded in $\tau \in \Omega$. Since $U_{1}$ is written as (5.29), it follows from Lemma 5.2, (5.9) and (U), that for $|\gamma| \leqq k+1$,

$$
\sup _{\tau \in \Omega}\left\|\left(v^{-1} D\right)^{r} U_{1}\right\|<\infty .
$$

This together with (5.34) implies

$$
\sup _{\tau \in \Omega}\left\|X_{k+1} A(\tau)^{k+1} U_{1}\right\|<\infty .
$$

Now, by induction we have

$$
\left.\left.\left[U_{1}, A(\tau)^{k+1}\right]=\sum_{j=0}^{k} C_{j} A(\tau)^{j}\left[\cdots\left[U_{1}, A(\tau)\right], A(\tau)\right] \cdots\right], A(\tau)\right],
$$

where the multiple commutators are $(k+1-j)$ fold commutators, and each $C_{j}$ is independent of $\tau \in \Omega$. By (5.3) we see that

$$
\left.\left.\sup _{\tau \in \Omega} \|\left(v^{-1} D\right)^{2}\left[\cdots\left[U_{1}, A(\tau)\right], \Lambda(\tau)\right] \cdots\right], .1(\tau)\right] \|<\infty,
$$

in the same way as (5.35), where $|\gamma| \leqq k+1$ and the multiple commutator is $(k+1-j)$ fold commutator $(0 \leqq j \leqq k)$. Thus, by (5.34) we obtain,

$$
\sup _{\tau \in \Omega}\left\|X_{k+1}\left[U_{1}, A(\tau)^{k+1}\right]\right\|<\infty .
$$

Therefore, we get (5.30) by (5.36), (5.38) and interpolation. This completes the proof of Proposition 3.1 (i).

Proof of Proposition 3.1 (ii). We denote by $T(\tau)$ the self-adjoint operator $-i \omega \cdot \nabla_{y}$ in $\mathscr{H}$ for each $\tau=(v, \omega) \in \Omega$. Then, for each $\tau=(v, \omega) \in \Omega$, we have

$$
i[T(\tau), A(\tau)]=\left(n_{a} v\right)^{-1} T(\tau)+1 \quad \text { on } \mathcal{S} .
$$

Hence, it follows that $T(\tau) \in S_{m}(A(\tau))$ and

$$
\sup _{\tau \in \Omega}\|T(\tau)\|_{S_{m}(A(\tau))}<\infty,
$$

for any integer $m \geqq 0$, and that

$$
\chi(T(\tau)) i[T(\tau), A(\tau)] \chi(T(\tau)) \geqq(1 / 2) \chi(T(\tau))^{2}
$$

for all $\tau \in \Omega$, where $\chi$ is the same as in Lemma 5.1. Now we set $r(\tau, z):=$ $(T(\tau)-z)^{-1}$ for $\operatorname{Im} z \neq 0$ and write 


$$
\begin{aligned}
{[R(\tau, i \varepsilon)-r(\tau, i \varepsilon)]=} & -v^{-1} R(\tau, i \varepsilon) r(\tau, i \varepsilon)\left(H_{0}-\lambda_{\alpha}\right) \\
& -v^{-1} R(\tau, i \varepsilon) V r(\tau, i \varepsilon) .
\end{aligned}
$$

Since $\left\|\langle A(\tau)\rangle^{2}\left(H_{0}-\lambda_{\alpha}\right)\right\|_{\boldsymbol{B}\left(H_{2}^{4}, L^{2}\right)}$ and $\left\|\langle A(\tau)\rangle^{2}\right\|_{\boldsymbol{B}\left(H_{2}^{4}, L^{2}\right)}$ are uniformly bounded in $\tau \in \Omega$, for the proof of Proposition 3.1 (ii) we have only to prove the following:

$$
\begin{aligned}
& \sup _{\tau \in \Omega, 0<\varepsilon<1}\left\|X_{2} R(\tau, i \varepsilon) r(\tau, i \varepsilon)\langle A(\tau)\rangle^{-2}\right\|<\infty, \\
& \sup _{\tau \in \Omega, 0<\varepsilon<1}\left\|X_{2} R(\tau, i \varepsilon) \operatorname{Vr}(\tau, i \varepsilon)\langle A(\tau)\rangle^{-2}\right\|<\infty .
\end{aligned}
$$

We only prove (5.43). (5.42) is proved similary. By the relation

$$
R(\tau, i \varepsilon)=R(\tau, i)+(i \varepsilon-i) R(\tau, i)^{2}+(i \varepsilon-i)^{2} R(\tau, i)^{2} R(\tau, i \varepsilon),
$$

the proof of (5.13) is reduced to proving the uniform boundedness of the following norms :

$$
\left\|X_{2} R(\tau, i)^{m}\langle A(\tau)\rangle\right\|\left\|\langle A(\tau)\rangle^{-1} V^{\gamma}\langle A(\tau)\rangle\right\|\left\|\langle A(\tau)\rangle^{-1} r(\tau, i \varepsilon)\langle A(\tau)\rangle^{-1}\right\|
$$

for $m=1,2$ and

$$
\left\|X_{2} R(\tau, i)^{2}\langle A(\tau)\rangle^{2}\right\|\left\|\langle A(\tau)\rangle^{-2} R(\tau, i \varepsilon) V r(\tau, i \varepsilon)\langle A(\tau)\rangle^{-2}\right\| .
$$

Both of the first factors in (5.44) and (5.45) are uniformly bounded in $\tau$ in the same way as (5.30). The second factor in (5.44) is uniformly bounded by (U), By (5.40), (5.41) and Theorem 4.2 with $d=1, K_{1}=T(\tau), A=A(\tau)$ we can prove the uniform boundedness of the last factor in (5.44), and by (5.40), (5.41), Lemma 5.1 and Theorem 4.2 with $d=2, K_{1}=L(\tau), K_{2}=T(\tau), W_{1}=V$ and $A=A(\tau)$ we can prove the uniform boundedness of the second factor in (5.45). Hence, we have shown that (5.44) and (5.45) are uniformly bounded. 'This completes the proof of Proposition 3.1 (ii).

\section{Appendix}

In this appendix we will prove Proposition 2.4 by supplementing the proof of Proposition 2 in [AS]. Throughout the discussion in this appendix we always assume $(\mathrm{V})_{0}$ and $(\mathrm{Z})$. Then $\kappa:=-\max \left\{\mu ; \mu \in \bigcup_{c \in A_{2}} \sigma_{P}\left(h_{c}\right)\right\}$ is strictly positive, where $\sigma_{P}\left(h_{c}\right)$ denotes the point spectrum of $h_{c}$. For $c=\{(i, j), k\} \in \boldsymbol{A}_{2}$, we denote by $V_{c}$ the potential $V_{\imath j}\left(x_{c}\right)$. Fix arbitrary $r_{0} \gg 1$ and let $\chi_{1}(\lambda)$ and $\chi_{0}(\lambda)$ be the characteristic functions for $\left[0, r_{0}+(\kappa / 4)\right]$ and $I_{0}:=\left[r_{0}-(\kappa / 4), r_{0}+\right.$ $(\kappa / 4)]$, respectively. Recall that $a \in A_{2}$ is the 2-cluster decomposition associated with the initial channel $\alpha \in \Gamma_{2}: D(\alpha)=a$. We put off the proof of the following lemma.

Lemma A-1. Let $c, d \in \boldsymbol{A}_{2}$ with $d \neq a$. Then the operator 


$$
F_{c d}(z):=\chi_{1}\left(T_{c}\right)\left\langle x_{c}\right\rangle V_{c} R(z) V_{d}\left\langle x_{a}\right\rangle^{-2 \delta}\left\langle y_{a}\right\rangle^{\delta-(1 / 2)}
$$

has the norm limits $F_{c d}(\lambda \pm i 0):=\lim _{\varepsilon \downarrow 0} F_{c d}(\lambda \pm i \varepsilon)$ in $\boldsymbol{B}(\mathscr{H})$ uniformly in $\lambda \in I_{0}$ (see (1.2) for $\delta$ ).

For the proof of the next lemma, see, for example, [GM] (Proposition 2.2).

Lemma A-2. The operator $\left.Z_{0}(\lambda)\left\langle x_{c}\right\rangle^{-1}, \lambda\right\rangle 0$, defined on $\mathcal{S}$, extends to a bounded operator from $\mathcal{H}$ to $L^{2}\left(S^{2 N-1}\right)$ for each $c \in \boldsymbol{A}_{2}$, and $Z_{0}(\lambda)\left\langle x_{c}\right\rangle^{-1} f, f \in \mathscr{H}$, is strongly continuous in $\lambda>0$.

Proof of Proposition 2.4. By $\chi_{0}\left(H_{0}\right) \chi_{1}\left(T_{c}\right)=\chi_{0}\left(H_{0}\right)$ for any $c \in A_{2}, Z_{0}(\lambda) \chi_{0}\left(H_{0}\right)$ $=\chi_{0}(\lambda) Z_{0}(\lambda)$ and Lemmas A-1, A-2, we get the norm limit

$$
\begin{aligned}
Z_{0}(\lambda) & {\left[-I_{a}+V R(\lambda+i 0) I_{a}\right] J_{\alpha} Z_{a}(\lambda)^{*} } \\
= & \lim _{\varepsilon \downarrow 0} Z_{0}(\lambda)\left[-I_{a}+V R(\lambda+i \varepsilon) I_{a}\right] J_{\alpha} Z_{a}(\lambda)^{*} \\
= & -Z_{0}(\lambda)\left\langle x_{a}\right\rangle^{-1}\left\langle x_{a}\right\rangle I_{a} J_{\alpha} Z_{\alpha}(\lambda)^{*} \\
& +\sum_{\substack{c \\
d \neq a}} Z_{0}(\lambda)\left\langle x_{c}\right\rangle^{-1} F_{c d}(\lambda+i 0)\left\langle x_{a}\right\rangle^{2 \delta}\left\langle y_{a}\right\rangle^{-\delta+(1 / 2)} J_{\alpha} Z_{a}(\lambda)^{*}
\end{aligned}
$$

for $\lambda \in I_{0}$. Here we note that $I_{a}\left\langle x_{a}\right\rangle J_{\alpha} Z_{a}(\lambda)^{*},\left\langle x_{a}\right\rangle^{2 \delta}\left\langle y_{a}\right\rangle^{-\delta+(1 / 2)} J_{\alpha} Z_{\alpha}(\lambda)^{*}$ is continuous in $\lambda \in I_{0}$ w.r.t. the Hilbert-Schmidt norm. Thus, by noting that $Z_{0}(\lambda)\left\langle x_{c}\right\rangle^{-1}$ is strongly continuous in $\lambda$ and $F_{c d}(\lambda+i 0)$ is norm continuous in $\lambda \in I_{0}$, we see that

$$
2 \pi i Z_{0}(\lambda)\left[-I_{a}+V R(\lambda+i 0) I_{a}\right] J_{\alpha} Z_{\alpha}(\lambda)^{*}
$$

is continuous in $\lambda \in I_{0}$ with respect to the Hilbert-Schmidt norm as an operator from $L^{2}\left(S^{N-1}\right)$ to $L^{2}\left(S^{2 N-1}\right)$. Furthermore, in almost the same way as in the 2 -body case, we can see that the above operator is equal to $T_{\alpha \rightarrow 0}(\lambda)$. Thus the integral kernel of $T_{\alpha \rightarrow 0}(\lambda)$ is given by

$$
T_{\alpha \rightarrow 0}(\lambda, \theta, \omega)=2 \pi i C_{n}(\lambda)\left(Z_{0}(\lambda) f(\lambda, \omega, \cdot)\right)(\theta),
$$

where

$$
f(\lambda, \omega, X)=\left(\left[-I_{a}+V R(\lambda+i 0) I_{a}\right] \psi_{\alpha} e^{i\left(2 n_{a}\left(\lambda-\lambda_{a}\right)\right)^{1 / 2} \omega \cdot y_{a}}\right)(X),
$$

$\mathrm{X}=\left(x_{a}, y_{a}\right)$. Moreover, we can also see that $T_{\alpha \rightarrow 0}(\lambda, \cdot, \omega)$ is $L^{2}\left(S^{2 N-1}\right)$-valued continuous function in $\lambda \in I_{0}$ and $\omega \in S^{N-1}$. Thus we have proved Proposition 2.4, accepting Lemma A-1.

Finally we prove Lemma A-1. The proof is given for the + case and divided into several steps. From now on, $c$ and $d$ denote 2-cluster decompositions.

Step 1. Let us begin with introducing notations. For $c \in \boldsymbol{A}_{2}$ we denote by 
$A_{c}, B_{c}$ and $C_{c}$ the multiplication operators $\left|V_{c}\left(x_{c}\right)\right|^{1 / 2},\left|V_{c}\left(x_{c}\right)\right|^{1 / 2} \operatorname{sgn} V_{c}\left(x_{c}\right)$ and $\left\langle y_{c}\right\rangle^{-\delta / 4}$, respectively. New Hilbert spaces $\mathscr{H}_{1}$ and $\mathscr{H}_{2}$ are defined by

$$
\mathscr{H}_{1}:=\mathscr{H} \oplus \mathscr{H} \oplus \mathscr{H}, \quad \mathscr{K}_{2}:=\mathscr{H}_{1} \oplus \mathscr{H}_{1} .
$$

We associate each $\mathscr{H}$ in $\mathscr{H}_{1}$ with each 2-cluster decomposition. Each operator $Q$ on $\mathscr{H}_{1}$ is an operator valued $3 \times 3$ matrix with the $c-d$ component $Q^{c d}(c, d$ $\in \boldsymbol{A}_{2}$ ), which is an operator on $\mathscr{H}$. We label two copies of $\mathscr{H}_{1}$ in $\mathscr{H}_{2} 0$ and 1 , respectively, and denote by $R_{\imath j}(0 \leqq i, j \leqq 1)$ the $i-j$ component of an operator $R$ on $\mathscr{H}_{2}$. Of course, each $R_{2 j}$ is an operator on $\mathscr{H}_{1}$.

We introduce several operators.

$$
\begin{aligned}
Y(z) \in \boldsymbol{B}\left(\mathscr{H}_{1}\right): \quad Y(z)^{c d}=B_{c} R(z) C_{d} \\
G(z) \in \boldsymbol{B}\left(\mathscr{H}_{1}\right): \quad G(z)^{c d}=B_{c} E_{c} R_{c}(z) C_{d} \delta_{c d}, \\
J(z)=(I d, G(z)) \in \boldsymbol{B}\left(\mathscr{H}_{2}, \mathscr{H}_{1}\right) \\
K(z)={ }^{t}\left(K_{0}(z), K_{1}(z)\right) \in \boldsymbol{B}\left(\mathscr{H}_{1}, \mathscr{H}_{2}\right): \\
\quad K_{0}(z)^{c d}=B_{c}\left(\mathrm{Id}-E_{c}\right) R_{c}(z) C_{d}, \quad K_{1}(z)^{c d}=C_{c}{ }^{-1} E_{c} C_{d} . \\
D_{00}(z) \in \boldsymbol{B}\left(\mathscr{H}_{1}\right): \quad D_{00}(z)^{c d}=B_{c}\left(\mathrm{Id}-E_{c}\right) R_{c}(z) A_{d}\left(\mathrm{Id}-\delta_{c d}\right), \\
D_{01}(z)=D_{00}(z) G(z) \in \boldsymbol{B}\left(\mathscr{H}_{1}\right), \\
D_{10}(z) \in \boldsymbol{B}\left(\mathscr{H}_{1}\right): \quad D_{10}(z)^{c d}:=C_{c}{ }^{-1} E_{c} A_{d}\left(\mathrm{Id}-\delta_{c d}\right), \\
D_{11}(z)=D_{10}(z) G(z) \in \boldsymbol{B}\left(\mathscr{H}_{1}\right), \\
D(z) \in \boldsymbol{B}\left(\mathscr{H}_{2}\right): D(z)_{\imath j}=D_{\imath j}(z) . \\
N \in \boldsymbol{B}\left(\mathscr{H}_{2}\right): N_{10}=D_{10}(z), \quad N_{\imath j}=0((i, j) \neq(1,0)), \\
W(z)=(I d-N)(D(z)-N) \in \boldsymbol{B}\left(\mathscr{H}_{2}\right) .
\end{aligned}
$$

Here $R_{c}(z):=\left(H_{c}-z\right)^{-1}, E_{c}:=P^{P}\left(h_{c}\right) \otimes \mathrm{Id}, P^{p}\left(h_{c}\right)$ being the orthogonal projection onto the subspace spanned by eigenvectors of $h_{c}$, and $\delta_{c d}$ is Kronecker's delta. For each $z \in \boldsymbol{C} \backslash \boldsymbol{R}, I d+W(z)$ has a bounded inverse and $Y(z)$ can be written as ([AS], p. 1572)

$$
Y(z)=J(z)(\operatorname{Id}+W(z))^{-1}(\operatorname{Id}-N) K(z) .
$$

Step 2. $W(z)$ has the following properties: (i) $W(z), z \in \boldsymbol{C} \backslash \boldsymbol{R}$, is a compact operator and has the norm limit $W(\lambda+i 0):=\lim _{\varepsilon \downarrow 0} W(\lambda+i \varepsilon)$ in $\boldsymbol{B}\left(\mathscr{K}_{2}\right)$ uniformly on any compact set in $(0, \infty)$, (ii) There exists a closed null set $e_{0}$ in $(0, \infty)$ such that $I d+W(\lambda+i 0)$ is invertible in $\lambda \in(0, \infty) \backslash e_{0}$.

We will show that $e_{0}$ is a bounded set by proving

$$
\|W(\lambda+i 0)\| \longrightarrow 0 \quad \text { as } \lambda \rightarrow \infty .
$$


The following lemma is important for the proof of (A5).

Lemma A-3. (i) ([GM], Proposition (2.4), [Ha], Corollary 5.5) Let $\delta>1$ and $R_{0}(z):=\left(H_{0}-z\right)^{-1}$. Then the norm limit

$$
\left\langle x_{c}\right\rangle^{-\delta} R_{0}(\lambda+i 0)\left\langle x_{d}\right\rangle^{-\delta}=\lim _{\varepsilon \downarrow 0}\left\langle x_{c}\right\rangle^{-\delta} R_{0}(\lambda+i \varepsilon)\left\langle x_{d}\right\rangle^{-\delta}
$$

exists in $\boldsymbol{B}(\mathscr{H})$ uniformly for $\lambda$ in any compact set in $\boldsymbol{R}$ for any $c, d$ and, moreover, we have

$$
\lim _{\lambda \rightarrow \infty}\left\|\left\langle x_{c}\right\rangle^{-\hat{o}} R_{0}(\lambda+i 0)\left\langle x_{d}\right\rangle^{-\delta}\right\|=0 \quad \text { for } c \neq d .
$$

(ii) (cf. [AJS], Lemma 16.15) The norm limit

$$
B_{c}\left(\mathrm{Id}-E_{c}\right) R_{c}(\lambda+i 0) A_{c}=\lim _{\varepsilon \downarrow 0} B_{c}\left(\mathrm{Id}-E_{c}\right) R_{c}(\lambda+i \varepsilon) A_{c}
$$

exists in $\boldsymbol{B}(\mathscr{H})$ uniformly for $\lambda$ in $\boldsymbol{R}$, and

$$
\sup _{\lambda \in R}\left\|B_{c}\left(\mathrm{Id}-E_{c}\right) R_{c}(\lambda+i 0) A_{c}\right\|<\infty
$$

for any $c$.

For the proofs, see [GM], [Ha] and [AJS].

In order to prove (A5), we will show that each $D_{\imath j}(\lambda+i \varepsilon), 0 \leqq i, j \leqq 1$, has the norm limit $D_{i j}(\lambda+i 0)$ in $\boldsymbol{B}\left(\mathscr{K}_{1}\right)$ as $\varepsilon \downarrow 0$ and all norms $\left\|D_{00}(\lambda+i 0)\right\|$, $\left\|D_{01}(\lambda+i 0)\right\|$ and $\left\|D_{11}(\lambda+i 0)\right\|$ go to zero as $\lambda \rightarrow \infty$. Then the relation

yields (A5).

$$
W(\lambda+i 0)=(\operatorname{Id}-N)(D(\lambda+i 0)-N)
$$

By the resolvent equation, we have

$$
\begin{aligned}
& B_{c}\left(\mathrm{Id}-E_{c}\right) R_{c}(\lambda+i \varepsilon) A_{d} \\
& \quad=-B_{c} E_{c} R_{0}(\lambda+i \varepsilon) A_{d}+\left[\mathrm{Id}-B_{c}\left(\mathrm{Id}-E_{c}\right) R_{c}(\lambda+i \varepsilon) A_{c}\right] B_{c} R_{0}(\lambda+i \varepsilon) A_{d} .
\end{aligned}
$$

For $c \neq d$, by $(\mathrm{V})_{\iota}$, Lemmas 2.2 and $\mathrm{A}-3$, the R. H. S. has the norm limit in $\boldsymbol{B}(\mathscr{H})$ as $\varepsilon \downarrow 0$ and the norm limit goes to zero as $\lambda \rightarrow \infty$. This proves the existence of the norm limit $D_{00}(\lambda+i 0)$ and

$$
\lim _{\lambda \rightarrow \infty}\left\|D_{00}(\lambda+i 0)\right\|=0 \text {. }
$$

To prove the existence of $D_{01}(\lambda+i 0)$ and

$$
\lim _{\lambda \rightarrow \infty}\left\|D_{01}(\lambda+i 0)\right\|=0 \text {. }
$$

we must estimate the following operators (see (A6))

$$
\begin{aligned}
& -B_{c} E_{c} R_{0}(\lambda+i \varepsilon) V_{d} R_{d}(\lambda+i \varepsilon) E_{d} C_{d} \\
& \quad+\left[\mathrm{Id}-B_{c}\left(\mathrm{Id}-E_{c}\right) R_{c}(\lambda+i \varepsilon) A_{c}\right] B_{c} R_{0}(\lambda+i \varepsilon) V_{d} R_{d}(\lambda+i \varepsilon) E_{d} C_{d}
\end{aligned}
$$


for $c \neq d$. The norm of the operator in $[\cdots]$ is uniformly bounded in $\varepsilon \in(0,1]$ and $\lambda \gg 1$ by Lemma A-3 (ii). Furthermore, by the resolvent equation, we have

$$
\begin{aligned}
& B_{c} R_{0}(\lambda+i \varepsilon) V_{d} R_{d}(\lambda+i \varepsilon) E_{d} C_{d} \\
& \quad=B_{c} R_{0}(\lambda+i \varepsilon) E_{d} C_{d}-B_{c} E_{d} R_{d}(\lambda+i \varepsilon) E_{d} C_{d} .
\end{aligned}
$$

Then, the first term and the second have norm limits in $\boldsymbol{B}(\mathscr{H})$ and the norms of these limits go to zero as $\lambda \rightarrow \infty$ by Lemma A-3 (i) and the well known fact (cf. [GM], Proposition (2.3)) :

$$
\lim _{\lambda \rightarrow \infty}\left\|\left\langle y_{d}\right\rangle^{-1}\left(-\Delta_{y_{d}}-\lambda-i 0\right)^{-1}\left\langle y_{d}\right\rangle^{-1}\right\|=0 .
$$

The first term in (A9) has the norm limit and the norm of this limit goes to zero as $\lambda \rightarrow \infty$ in the same way as above. Thus we have proved (A8). By (A10) and $C_{c}^{-1} E_{c} A_{d} B_{d} C_{d}^{-1} E_{d} \in \boldsymbol{B}(\mathcal{H})$ for $c \pm d, C_{c}{ }^{-1} E_{c} A_{d} B_{d} E_{d} R_{d}(\lambda+i \varepsilon) C_{d}$ has the norm limit in $\boldsymbol{B}(\mathscr{H})$ as $\varepsilon \downarrow 0$ and the limit goes to zero as $\lambda \rightarrow \infty$ for $c \neq d$. This implies the existence of $D_{11}(\lambda+i 0)$ and

$$
\lim _{\lambda \rightarrow \infty} \| D_{11}(\lambda+i 0)||=0 \text {. }
$$

Thus we have proved (A5).

Step 3. Finally we prove Lemma A-1. We assume $r_{0}-(\kappa / 4)>\sup e_{0}$. Since

$$
\begin{aligned}
F_{c d}(z):= & \chi_{1}\left(T_{c}\right)\left\langle x_{c}\right\rangle A_{c} \\
& \times\left(J(z)(I d+W(z))^{-1}(\operatorname{Id}-N) K(z)\right)^{c d} V_{d} C_{d}{ }^{-1}\left\langle x_{a}\right\rangle^{-2 \tilde{o}}\left\langle y_{a}\right\rangle^{\hat{o}-(1 / 2)},
\end{aligned}
$$

it suffices to show that

$$
\chi_{1}\left(T_{c}\right)\left\langle x_{c}\right\rangle A_{c} G(\lambda+i \varepsilon)^{c f}=\left\langle x_{c}\right\rangle V_{c} E_{c} \chi_{1}\left(T_{c}\right) R_{c}(\lambda+i \varepsilon) C_{c} \delta_{c f}
$$

and

$$
\begin{aligned}
& K_{0}(\lambda+i \varepsilon)^{g d} V_{d} C_{d}{ }^{-1}\left\langle x_{a}\right\rangle^{-2 \delta}\left\langle y_{a}\right\rangle^{\tilde{\delta}-(1 / 2)} \\
& \quad=B_{g}\left(\mathrm{Id}-E_{g}\right) R_{g}(\lambda+i \varepsilon)\left\langle x_{a}\right\rangle^{-\delta} V_{d}\left\langle x_{a}\right\rangle^{-\hat{o}}\left\langle y_{a}\right\rangle^{\hat{\delta}-(1 / 2)},
\end{aligned}
$$

have norm limits in $\boldsymbol{B}(\mathscr{H})$ as $\varepsilon \downarrow 0$ uniformly in $\lambda \in I_{0}$ for any $c, f, g, d \in \mathbb{A}_{2}$ with $d \neq a$. By the choice of $\kappa$ and $I_{0}$, the first has norm limit. Since $B_{g}\left(\mathrm{Id}-E_{g}\right) R_{g}(\lambda+i \varepsilon)\left\langle x_{a}\right\rangle^{-\hat{o}}=B_{g}\left(\mathrm{Id}-E_{g}\right) R_{0}(\lambda+i \varepsilon)\left\langle x_{a}\right\rangle^{-\hat{o}}-B_{g}\left(\mathrm{Id}-E_{g}\right) R_{g}(\lambda+i \varepsilon) \times$ $A_{g} B_{g} R_{0}(\lambda+i \varepsilon)\left\langle x_{a}\right\rangle^{-\delta}$ and $V_{d}\left\langle x_{a}\right\rangle^{-\delta}\left\langle y_{a}\right\rangle^{\delta-(1 / 2)}$ is bounded by $a \neq d$, we see that the second has the norm limit by Lemma A-3. This completes the proof of Lemma A-1.

\section{References}

[Ag] Agmon, S., Lectures on exponential decay of solutions of second order elliptic equations, Princeton, Princeton University Press, 1982.

[ABG] Amrein, W.O., Berthier, A.M. and Georgescu, V., On Mourre's approach to spectral theory, Helv. Phys. Acta., 62 (1989), 1-20. 
[AJS] Amrein, W.O., Jauch, J.M. and Sinha, K. B., Scattering theory in quantum mechanics, Benjamin, Reading, 1977.

[APS] Amrein, W. O., Pearson, D. B. and Sinha, K. B., Bounds on the total scattering cross-section for $N$-body systems, Nuovo Cimento, 52 (1979), 115-131.

[AS] Amrein, W.O. and Sinha, K. B., On three-body scattering cross sections, J. Phys. A: Math. Gen., 15 (1982), 1567-1586.

[CFKS] Cycon, H. L., Froese, R.G., Kirsch, W. and Simon, B., Schrödinger operators, Springer, Berlin, Heidelberg, New York, 1987.

[E] Enss, V., "Quantum scattering theory of two-and three-body systems with potentials of short and long range" in Schrödinger operators, ed. S. Graffi, Lecture Notes in Mathematics, 1159, Springer, Berlin, Heidelberg, New York, 1985.

[ES] Enss, V. and Simon, B.. Finite total cross sections in nonrelativistic quantum mechanics, Comm. Math. Phys., 76 (1980), 177-209.

[GM] Ginibre, J. and Moulin, M., Hilbert space approach to the quantum mechanical three body problem, Ann. Inst. H. Poincaré, A21 (1974), 97-145.

[Ha] Hagedorn, G. A., Born series for (2 cluster) $\rightarrow$ (2 cluster) Scattering of two, three, and four particle Schrödinger operators, Comm. Math. Phys., 66 (1979), 77-94.

[I] Ito, H. T., Charge transfer model and (2 cluster $) \rightarrow(2$ cluster $)$ three-body scattering, to appear in J. Math. Kyoto Univ.

[IT] Ito, H. T. and Tamura, H., Semi-classical asymptotics for total scattering cross sections of 3-body systems, J. Math. Kyoto Univ., 32 (1992), 533-555.

[J] Jensen, A., Propagation estimates for Schrödinger-type operators, Trans. AMS, 291 (1985), 129-144.

[JMP] Jensen, A., Mourre, E. and Perry, P.A., Multiple commutator estimates and resolvent smoothness in quantum scattering theory, Ann. Inst. H. Poincaré A, 41 (1984), 207-225.

[M] Mourre, E., Absence of singular continuous spectrum for certain selfadjoint operators, Comm. Math. Phys., 78 (1981), 391-408.

[PSS] Perry, P., Sigal, I. M. and Simon, B., Spectral analysis of $N$-body Schrödinger operators, Ann. Math., 114 (1981), 519-567.

[RS] Reed, M. and Simon, B., Methods of modern mathematical physics, III, IV, Academic Press, 1978, 1979.

[T] Tamura,, H. Principle of limiting absorption for $N$-body Schrödinger operators, -a remark on the commutator methods, Lett. in Math. Phys., 17 (1989), 31-36. 